\title{
THE VIETORIS FUNCTOR AND MODAL OPERATORS ON RINGS OF CONTINUOUS FUNCTIONS
}

\author{
G. BEZHANISHVILI, L. CARAI, P. J. MORANDI
}

\begin{abstract}
We introduce an endofunctor $\mathscr{H}$ on the category $\boldsymbol{b} \boldsymbol{a} \boldsymbol{\ell}$ of bounded archimedean $\ell$-algebras and show that there is a dual adjunction between the category $\operatorname{Alg}(\mathscr{H})$ of algebras for $\mathscr{H}$ and the category Coalg $(\mathscr{V})$ of coalgebras for the Vietoris endofunctor $\mathscr{V}$ on the category of compact Hausdorff spaces. We also introduce an endofunctor $\mathscr{H}^{u}$ on the reflective subcategory of $\boldsymbol{b} \boldsymbol{a} \boldsymbol{\ell}$ consisting of uniformly complete objects of $\boldsymbol{b} \boldsymbol{a} \boldsymbol{\ell}$ and show that Gelfand duality lifts to a dual equivalence between $\operatorname{Alg}\left(\mathscr{H}^{u}\right)$ and $\operatorname{Coalg}(\mathscr{V})$. On the one hand, this generalizes a result of 1, 19] for the category of coalgebras of the Vietoris endofunctor on the category of Stone spaces. On the other hand, it yields an alternate proof of a recent result of [7].
\end{abstract}

\section{INTRODUCTION}

It is a well-known result in modal logic that the category MA of modal algebras is dually equivalent to the category DF of descriptive frames. This result has its origins in the work of Jónsson and Tarski [18, which is why it is sometimes referred to as Jónsson-Tarski duality. In its present form it was established by Esakia [13] and Goldblatt [14] (but see also Halmos [15]).

A descriptive frame is a Stone space (compact Hausdorff zero-dimensional space) $X$ equipped with a binary relation $R$ that is continuous, meaning that the corresponding map $\rho_{R}: X \rightarrow \mathscr{V}(X)$ into the Vietoris space of $X$, given by

$$
\rho_{R}(x)=R[x]=\{y \mid x R y\},
$$

is a well-defined continuous map. In fact, DF is isomorphic to the category Coalg $(\mathscr{V})$ of coalgebras for the Vietoris endofunctor $\mathscr{V}$ on the category Stone of Stone spaces. Abramsky [1] and Kupke, Kurz, and Venema 19 defined the dual endofunctor $\mathscr{H}$ on the category BA of boolean algebras. They showed that the category $\operatorname{Alg}(\mathscr{H})$ of algebras for $\mathscr{H}$ is isomorphic to MA, and proved that Stone duality between BA and Stone lifts to a dual equivalence between $\operatorname{Alg}(\mathscr{H})$ and $\operatorname{Coalg}(\mathscr{V})$. This yields an elegant new proof of the Esakia-Goldblatt theorem that MA is dually equivalent to DF.

Let KHaus be the category of compact Hausdorff spaces and continuous maps. Then Stone is a full subcategory of KHaus. There are several generalizations of Stone duality to KHaus. To outline one such generalization, we point out that in Stone duality we work with the boolean algebra of clopens, which correspond to continuous characteristic functions. Since

2010 Mathematics Subject Classification. 54B20; 54D30; 54C30; 06F25; 13J25; 06E15; 06E25; 03B45.

Key words and phrases. Compact Hausdorff space, continuous relation, coalgebra, bounded archimedean $\ell$-algebra, uniform completeness, Gelfand duality, Stone space, modal algebra, Jónsson-Tarski duality. 
an arbitrary compact Hausdorff space $X$ does not have enough clopens, it is natural to work instead with the ring $C(X)$ of all continuous real-valued functions. This gives rise to the celebrated Gelfand duality between KHaus and the category of bounded archimedean $\ell$-algebras that in addition are uniformly complete (see Section 2.1 for details). Up to isomorphism, these are exactly the rings $C(X)$ for $X \in \mathrm{KHaus}$.

The Vietoris endofunctor $\mathscr{V}:$ Stone $\rightarrow$ Stone is the restriction of the Vietoris endofunctor $\mathscr{V}:$ KHaus $\rightarrow$ KHaus. We call a relation $R$ on $X \in$ KHaus continuous if $\rho_{R}: X \rightarrow$ $\mathscr{V}(X)$ is a well-defined continuous map. The pairs $(X, R)$, where $X \in$ KHaus and $R$ is a continuous relation on $R$, generalize descriptive frames. Following [7], we call such pairs compact Hausdorff frames, and denote the resulting category by KHF. Then DF is a full subcategory of KHF and the isomorphism between DF and the category of coalgebras for $\mathscr{V}:$ Stone $\rightarrow$ Stone extends to an isomorphism between KHF and the category of coalgebras for $\mathscr{V}:$ KHaus $\rightarrow$ KHaus.

Our aim is to generalize the endofunctor $\mathscr{H}: \mathrm{BA} \rightarrow \mathrm{BA}$ that is the algebraic counterpart of $\mathscr{V}:$ Stone $\rightarrow$ Stone to an endofunctor on the category $\boldsymbol{b} \boldsymbol{a} \boldsymbol{\ell}$ of bounded archimedean $\boldsymbol{\ell}$-algebras so that it is the algebraic counterpart of $\mathscr{V}:$ KHaus $\rightarrow$ KHaus. For this we need to overcome several obstacles. Firstly, the construction of $\mathscr{H}: \mathrm{BA} \rightarrow \mathrm{BA}$ utilizes the existence of free boolean algebras. However, as was shown in [6], free algebras on sets do not exist in $\boldsymbol{b} \boldsymbol{a} \boldsymbol{\ell}$. Instead we need to work with free algebras on weighted sets (see Section 2.2). Secondly, since KHaus is dually equivalent to the reflective subcategory $\boldsymbol{u b a} \boldsymbol{\ell}$ of $\boldsymbol{b} \boldsymbol{a} \boldsymbol{\ell}$ consisting of uniformly complete objects in $\boldsymbol{b} \boldsymbol{a} \boldsymbol{\ell}$, additional care is needed when transitioning from $\boldsymbol{b} \boldsymbol{a} \boldsymbol{\ell}$ to its subcategory $\boldsymbol{u b a \ell}$.

We first construct an endofunctor $\mathscr{H}: \boldsymbol{b} \boldsymbol{a} \boldsymbol{\ell} \rightarrow \boldsymbol{b} \boldsymbol{a} \boldsymbol{\ell}$ and prove that there is a dual adjunction between $\operatorname{Alg}(\mathscr{H})$ and $\operatorname{Coalg}(\mathscr{V})$. We next construct an endofunctor $\mathscr{H}^{u}: \boldsymbol{u b a} \boldsymbol{\ell} \rightarrow \boldsymbol{u b a} \boldsymbol{\ell}$ and prove that there is a dual equivalence between $\operatorname{Alg}\left(\mathscr{H}^{u}\right)$ and $\operatorname{Coalg}(\mathscr{V})$. As a result, we obtain a generalization of the result of Kupke, Kurz, and Venema [19] from Stone to KHaus.

In [7] we generalized the notion of a modal operator on a boolean algebra to that of a modal operator on $A \in \boldsymbol{b} \boldsymbol{a} \boldsymbol{\ell}$. This has resulted in the category $\boldsymbol{m} \boldsymbol{b a} \boldsymbol{\ell}$ of modal bounded archimedean $\ell$-algebras and its reflective subcategory muba $\ell$ consisting of uniformly complete objects. The main result of [7] establishes that there is a dual adjunction between $\boldsymbol{m b a} \boldsymbol{\ell}$ and KHF, which restricts to a dual equivalence between mubal and KHF. On the one hand this generalizes Gelfand duality, and on the other hand the duality between MA and DF. Here we show that $\operatorname{Alg}(\mathscr{H})$ is isomorphic to $\boldsymbol{m b a} \boldsymbol{\ell}$ and that $\operatorname{Alg}\left(\mathscr{H}^{u}\right)$ is isomorphic to $\boldsymbol{m u b a} \boldsymbol{\ell}$. From this the main result of [7] follows, and we arrive at the following diagram, where the hook arrows are embeddings of categories, the two-sided horizontal arrows are dual equivalences, and the vertical arrows are isomorphisms.

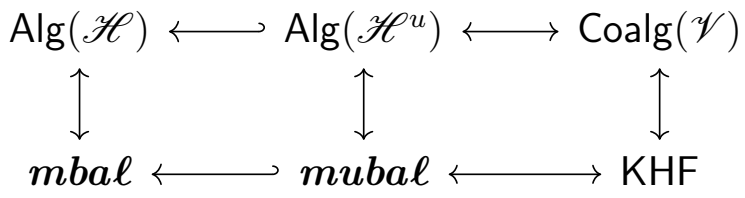

The paper is organized as follows. Section 2 provides the necessary background for the paper, including Gelfand duality, the construction of free objects in $\boldsymbol{b} \boldsymbol{a} \boldsymbol{\ell}$, and the definition 
of modal operators on objects of $\boldsymbol{b a} \boldsymbol{\ell}$. In Section 3 we introduce the endofunctor $\mathscr{H}$ on $\boldsymbol{b a} \boldsymbol{\ell}$, and in Section 4 we show that the category of algebras for $\mathscr{H}$ is isomorphic to $\boldsymbol{m b a} \boldsymbol{\ell}$. We relate $\mathscr{H}$ to the Vietoris functor $\mathscr{V}$ in Section 5 by showing that for $A \in \boldsymbol{b a} \boldsymbol{\ell}$, the Yosida space of $\mathscr{H}(A)$ is homeomorphic to the Vietoris space of the Yosida space of $A$. We prove our main result in Section 6, establishing a dual adjunction between $\operatorname{Alg}(\mathscr{H})$ and $\operatorname{Coalg}(\mathscr{V})$. We then introduce a reflective subcategory $\operatorname{Alg}^{u}(\mathscr{H})$ of $\operatorname{Alg}(\mathscr{H})$ and show that this dual adjunction restricts to a dual equivalence between $\operatorname{Alg}^{u}(\mathscr{H})$ and Coalg $(\mathscr{V})$. We also provide an alternate view of the category $\operatorname{Alg}^{u}(\mathscr{H})$ as $\operatorname{Alg}\left(\mathscr{H}^{u}\right)$. In Section 7 we derive the main result of [7], showing that there is a dual adjunction between $\boldsymbol{m b a} \boldsymbol{\ell}$ and KHF, which restricts to a dual equivalence between mubal and KHF. In Section 8 we show how the exclusion of the empty set from the construction of the Vietoris space results in the modification of the $\mathscr{H}$ functor to the functor $\mathscr{H}^{*}$ such that $\operatorname{Alg}\left(\mathscr{H}^{*}\right)$ is isomorphic to the full subcategory $\boldsymbol{m b a} \boldsymbol{\ell}^{\mathrm{D}}$ of those $(A, \square)$ where $\square$ corresponds to a serial relation. Finally, in Section 9 we relate our results to those of [19].

\section{Preliminaries}

In this section we provide the necessary background for the rest of the paper. In $\S 2.1$ we recall Gelfand duality, in $\S 2.2$ free objects in $\boldsymbol{b} \boldsymbol{a} \boldsymbol{\ell}$ over weighted sets, and finally in $\S 2.3$ modal operators on algebras in $\boldsymbol{b} \boldsymbol{a} \boldsymbol{\ell}$ and a generalization of Gelfand duality to this setting.

2.1. Gelfand duality. For basic facts about lattice-ordered rings and algebras we use Birkhoff's book [10, Ch. XIII and onwards] as our main reference. All rings we consider are assumed to be commutative and unital.

\section{Definition 2.1.}

(1) A ring $A$ with a partial order $\leq$ is a lattice-ordered ring, or an $\ell$-ring for short, provided $(A, \leq)$ is a lattice, $a \leq b$ implies $a+c \leq b+c$ for each $c$, and $0 \leq a, b$ implies $0 \leq a b$.

(2) An $\ell$-ring $A$ is an $\ell$-algebra if it is an $\mathbb{R}$-algebra and for each $0 \leq a \in A$ and $0 \leq r \in \mathbb{R}$ we have $0 \leq r \cdot a$.

(3) An $\ell$-ring $A$ is bounded if for each $a \in A$ there is $n \in \mathbb{N}$ such that $a \leq n \cdot 1$ (that is, 1 is a strong order unit).

(4) An $\ell$-ring $A$ is archimedean if for each $a, b \in A$, whenever $n \cdot a \leq b$ for each $n \in \mathbb{N}$, then $a \leq 0$.

(5) An $\ell$-algebra morphism $\alpha: A \rightarrow B$ is both an $\mathbb{R}$-algebra and lattice homomorphism. It is unital if $\alpha(1)=1$.

(6) Let $\boldsymbol{b} \boldsymbol{a} \boldsymbol{\ell}$ be the category of bounded archimedean $\ell$-algebras and unital $\ell$-algebra morphisms.

Let $A \in \boldsymbol{b a} \boldsymbol{\ell}$. For $a \in A$, define the absolute value of $a$ by

$$
|a|=a \vee(-a) \text {. }
$$

If we set the positive and negative parts of $a$ to be $a^{+}=a \vee 0$ and $a^{-}=(-a)^{+}$, then $a=a^{+}-a^{-}$and $|a|=a^{+}+a^{-}$. 
The norm of $a$ is defined by

$$
\|a\|=\inf \{r \in \mathbb{R}|| a \mid \leq r \cdot 1\} .
$$

If $X \in$ KHaus, then $C(X) \in \boldsymbol{b a} \boldsymbol{\ell}$ and the definition of the norm of $f \in C(X)$ coincides with the usual definition

$$
\|f\|=\sup \{|f(x)| \mid x \in X\} .
$$

If $\alpha: A \rightarrow B$ is a $\boldsymbol{b a} \boldsymbol{\ell}$-morphism, it is easy to see that $\|\alpha(a)\| \leq\|a\|, \alpha\left(a^{+}\right)=\alpha(a)^{+}$, and $\alpha(r)=r$ for all $r \in \mathbb{R}$, where we identify $r$ with $r \cdot 1$.

Definition 2.2. We call $A \in \boldsymbol{b a} \boldsymbol{\ell}$ uniformly complete if its norm is complete. Let $\boldsymbol{u} \boldsymbol{b a} \boldsymbol{\ell}$ be the full subcategory of $\boldsymbol{b} \boldsymbol{a} \boldsymbol{\ell}$ consisting of uniformly complete objects of $\boldsymbol{b} \boldsymbol{a} \boldsymbol{\ell}$.

Theorem 2.3 (Gelfand duality). There is a dual adjunction between bal and KHaus which restricts to a dual equivalence between ubal and KHaus.

We briefly describe the functors $\mathscr{C}:$ KHaus $\rightarrow \boldsymbol{b} \boldsymbol{a} \boldsymbol{\ell}$ and $\mathscr{Y}: \boldsymbol{b} \boldsymbol{a} \boldsymbol{\ell} \rightarrow$ KHaus establishing the dual adjunction of Theorem [2.3, for details see [8, Sec. 3] and the references therein. For a compact Hausdorff space $X$ let $\mathscr{C}(X):=C(X)$ be the ring of (necessarily bounded) continuous real-valued functions on $X$. For a continuous map $\varphi: X \rightarrow Y$ let $\mathscr{C}(\varphi)$ : $C(Y) \rightarrow C(X)$ be defined by $\mathscr{C}(\varphi)(f)=f \circ \varphi$ for each $f \in C(Y)$. Then $\mathscr{C}:$ KHaus $\rightarrow \boldsymbol{b a} \boldsymbol{\ell}$ is a well-defined contravariant functor.

For $A \in \boldsymbol{b a} \ell$, we recall that an ideal $I$ of $A$ is an $\ell$-ideal if $|a| \leq|b|$ and $b \in I$ imply $a \in I$. If $A \in \boldsymbol{b} \boldsymbol{a} \boldsymbol{\ell}$, then we can associate to $A$ a compact Hausdorff space as follows. Let $Y_{A}$ be the space of maximal $\ell$-ideals of $A$, whose closed sets are exactly sets of the form

$$
Z_{\ell}(I)=\left\{M \in Y_{A} \mid I \subseteq M\right\}
$$

where $I$ is an $\ell$-ideal of $A$. It follows from the work of Yosida [23] that $Y_{A} \in \mathrm{KHaus}$. As is customary, we refer to $Y_{A}$ as the Yosida space of $A$ and set $\mathscr{Y}(A)=Y_{A}$. For a morphism $\alpha$ in $\boldsymbol{b} \boldsymbol{a} \boldsymbol{\ell}$ we let $\mathscr{Y}(\alpha)=\alpha^{-1}$. Then $\mathscr{Y}: \boldsymbol{b} \boldsymbol{a} \boldsymbol{\ell} \rightarrow \mathrm{KHaus}$ is a well-defined contravariant functor, and the functors $\mathscr{C}$ and $\mathscr{Y}$ yield a dual adjunction between $\boldsymbol{b} \boldsymbol{a} \boldsymbol{\ell}$ and KHaus.

For $X \in$ KHaus we have that $\varepsilon_{X}: X \rightarrow Y_{C(X)}$ is a homeomorphism where

$$
\varepsilon_{X}(x)=\{f \in C(X) \mid f(x)=0\} .
$$

For $A \in \boldsymbol{b a} \boldsymbol{\ell}$ define $\zeta_{A}: A \rightarrow C\left(Y_{A}\right)$ by $\zeta_{A}(a)(M)=r$ where $r$ is the unique real number satisfying $a+M=r+M$. Then $\zeta_{A}$ is a monomorphism in $\boldsymbol{b} \boldsymbol{a} \boldsymbol{\ell}$ separating points of $Y_{A}$. Therefore, by the Stone-Weierstrass theorem, $\zeta_{A}: A \rightarrow C\left(Y_{A}\right)$ is the uniform completion of $A$. Thus, if $A$ is uniformly complete, then $\zeta_{A}$ is an isomorphism. Consequently, the contravariant adjunction restricts to a dual equivalence between $\boldsymbol{u} \boldsymbol{b a} \boldsymbol{\ell}$ and KHaus, yielding Gelfand duality. Another consequence of these considerations is the following well-known result.

Proposition 2.4. uba $\boldsymbol{\ell}$ is a full reflective subcategory of $\boldsymbol{b a \ell}$, and the reflector assigns to

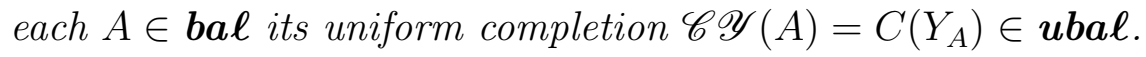


Remark 2.5. Since $\mathscr{C}$ and $\mathscr{Y}$ form a dual adjunction between $\boldsymbol{b a} \boldsymbol{\ell}$ and KHaus, the natural transformations $\zeta$ and $\varepsilon$ satisfy $\mathscr{Y}\left(\zeta_{A}\right) \circ \varepsilon_{Y_{A}}=1_{Y_{A}}$ and $\mathscr{C}\left(\varepsilon_{X}\right) \circ \zeta_{C(X)}=1_{C(X)}$ for each $A \in \boldsymbol{b a} \boldsymbol{\ell}$ and $X \in \mathrm{KHaus}$ by [20, Thm. IV.1.1]. Moreover, since $\varepsilon$ is a natural isomorphism, $\mathscr{Y}\left(\zeta_{A}\right)=\varepsilon_{Y_{A}}^{-1}$ and $\zeta_{C(X)}=\mathscr{C}\left(\varepsilon_{X}\right)^{-1}$.

If $A$ is an $\ell$-subalgebra of $B \in \boldsymbol{b a} \boldsymbol{\ell}$, we say $A$ is uniformly dense in $B$ if $A$ is dense in $B$ with respect to the topology induced by the norm on $B$. In the following lemma we collect several facts that will be used subsequently.

Lemma 2.6. [8, Lem. 2.9] Let $\alpha: A \rightarrow B$ be a ba $\boldsymbol{\ell}$-morphism.

(1) $\mathscr{Y}(\alpha)$ is onto iff $\alpha$ is 1-1 iff $\alpha$ is a monomorphism.

(2) $\mathscr{Y}(\alpha)$ is 1-1 iff $\alpha[A]$ is uniformly dense in $B$ iff $\alpha$ is an epimorphism.

(3) $\mathscr{Y}(\alpha)$ is a homeomorphism iff $\alpha$ is a bimorphism.

2.2. Free objects in $\boldsymbol{b a} \boldsymbol{\ell}$. By [6, Thm. 3.2], free objects on nonempty sets do not exist in $\boldsymbol{b a} \boldsymbol{\ell}$. To see this, observe that each $\boldsymbol{b a} \boldsymbol{\ell}$-morphism $\alpha: A \rightarrow B$ satisfies $\|\alpha(a)\| \leq\|a\|$ for each $a \in A$. Now suppose that a free object $F \in \boldsymbol{b a} \boldsymbol{\ell}$ exists on $X \neq \varnothing$. Let $f: X \rightarrow F$ be the corresponding map, let $x \in X$, and let $r \in \mathbb{R}$ satisfy $r>\|f(x)\|$. Define $g: X \rightarrow \mathbb{R}$ by $g(y)=r$ for each $y \in X$. Then there is a $\boldsymbol{b} \boldsymbol{a} \boldsymbol{\ell}$-morphism $\alpha: F \rightarrow \mathbb{R}$ with $\alpha \circ f=g$. Therefore, $\alpha(f(x))=g(x)=r$, violating the inequality $\|\alpha(f(x))\| \leq\|f(x)\|$. Taking this into account leads to the following notion.

Definition 2.7. [6, Def. 3.3]

- A weight function on a set $X$ is a function $w$ from $X$ into the nonnegative real numbers.

- A weighted set is a pair $(X, w)$ where $X$ is a set and $w$ is a weight function on $X$.

- A weighted set morphism $f:\left(X_{1}, w_{1}\right) \rightarrow\left(X_{2}, w_{2}\right)$ is a function $f: X_{1} \rightarrow X_{2}$ satisfying $w_{2}(f(x)) \leq w_{1}(x)$ for each $x \in X$.

There is a forgetful functor $U$ from $\boldsymbol{b} \boldsymbol{a} \boldsymbol{\ell}$ to the category of weighted sets that associates to each $A \in \boldsymbol{b a} \boldsymbol{\ell}$ the weighted set $(A,\|\cdot\|)$. By [6, Thm. 3.9], $U$ has a left adjoint, thus yielding the following theorem.

Theorem 2.8. Free objects in bal exist over weighted sets.

2.3. Modal operators on bounded archimedean $\ell$-algebras. In [7] the notion of a modal operator on $A \in \boldsymbol{b} \boldsymbol{a} \boldsymbol{\ell}$ was introduced, generalizing that of a modal operator on a boolean algebra. The motivating example comes from a continuous relation $R$ on a compact Hausdorff space $X$ (see Definition 2.11). If $R$ is serial (meaning $R[x] \neq \varnothing$ for each $x \in X$ ), then there is a natural definition of a modal operator $\square_{R}$ on $C(X)$, given by $\square_{R}(f)(x)=$ inf $f R[x]$ for each $x \in X$. It is straightforward to see that $\square_{R}$ preserves meet, 0,1 , addition by a scalar, and multiplication by a nonnegative scalar. If $R$ is not serial, then $\square_{R}$ needs to be redefined since $R[x]$ may be empty. For an arbitrary continuous relation we define $\square_{R}$ by

$$
\left(\square_{R} f\right)(x)=\left\{\begin{array}{cl}
\inf f R[x] & \text { if } R[x] \neq \varnothing \\
1 & \text { if } R[x]=\varnothing .
\end{array}\right.
$$


If $R$ is not serial, then $\square_{R} 0 \neq 0$, and the properties of $\square_{R}$ become more complicated. Looking carefully at those properties, we arrive at the following definition.

Definition 2.9. [7, Def. 3.10]

(1) Let $A \in \boldsymbol{b a} \boldsymbol{\ell}$. We say that a unary function $\square: A \rightarrow A$ is a modal operator on $A$ provided $\square$ satisfies the following axioms for each $a, b \in A$ and $r \in \mathbb{R}$ :

(M1) $\square(a \wedge b)=\square a \wedge \square b$.

(M2) $\square r=r+(1-r) \square 0$.

(M3) $\square\left(a^{+}\right)=(\square a)^{+}$.

(M4) $\square(a+r)=\square a+\square r-\square 0$.

(M5) $\square(r a)=(\square r)(\square a)$ provided $r \geq 0$.

(2) If $\square$ is a modal operator on $A \in \boldsymbol{b a} \boldsymbol{\ell}$, then we call the pair $(A, \square)$ a modal bounded archimedean $\ell$-algebra.

(3) Let $\boldsymbol{m b a} \boldsymbol{\ell}$ be the category of modal bounded archimedean $\ell$-algebras and unital $\ell$-algebra homomorphisms preserving $\square$.

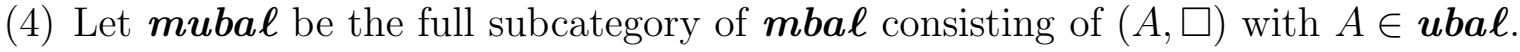

Remark 2.10. Let $(A, \square) \in \boldsymbol{m b a} \boldsymbol{\ell}$. Axiom (M1) implies that $\square$ is order preserving. From (M2) we have $\square 1=1$. Finally (M3) shows that if $0 \leq a$, then $\square a=(\square a)^{+}$, so $0 \leq \square a$. In particular, $0 \leq \square 0$.

Definition 2.11. [5, Sec. 2]

(1) A binary relation $R$ on a compact Hausdorff space $X$ is continuous if:

(a) $R[x]$ is closed for each $x \in X$.

(b) $F \subseteq X$ closed implies $R^{-1}[F]$ is closed.

(c) $U \subseteq X$ open implies $R^{-1}[U]$ is open.

(2) If $X$ is compact Hausdorff and $R$ is a continuous relation on $X$, then we call $(X, R)$ a compact Hausdorff frame.

(3) A bounded morphism (or p-morphism) between $(X, R)$ and $(Y, S)$ is a map $f: X \rightarrow Y$ satisfying $f(R[x])=S[f(x)]$ for each $x \in X$ (equivalently, $f^{-1}\left(S^{-1}[y]\right)=R^{-1}\left[f^{-1}(y)\right]$ for each $y \in Y)$.

(4) Let KHF be the category of compact Hausdorff frames and continuous bounded morphisms.

Theorem 2.12. [7, Thm. 5.3] There is a dual adjunction between mbal and KHF which restricts to a dual equivalence between mubal and KHF.

The functors establishing the adjunction of Theorem 2.12 extend those of Gelfand duality. If $(A, \square) \in \boldsymbol{m b a} \boldsymbol{\ell}$, define $R_{\square}$ on $Y_{A}$ by $x R_{\square} y$ if $0 \leq a \in y$ implies $\square a \in x$. Then $\mathscr{Y}(A, \square):=$ $\left(Y_{A}, R_{\square}\right) \in \mathrm{KHF}$. Going the other direction, if $(X, R) \in \mathrm{KHF}$, define $\square_{R}$ on $C(X)$ as above. Then $\mathscr{C}(X, R):=\left(C(X), \square_{R}\right) \in \boldsymbol{m b a}$.

\section{The ENDOFUnCTOR $\mathscr{H}: \boldsymbol{b} \boldsymbol{a} \boldsymbol{\ell} \rightarrow \boldsymbol{b} \boldsymbol{a} \boldsymbol{\ell}$}

In this section we define the endofunctor $\mathscr{H}$ on $\boldsymbol{b a} \boldsymbol{\ell}$. Let $A \in \boldsymbol{b a} \boldsymbol{\ell}$. Following [6, Def. 3.7], we call an $\ell$-ideal $I$ of $A$ archimedean if $A / I$ is archimedean (and hence $A / I \in \boldsymbol{b a} \ell$ ). 
Archimedean $\ell$-ideals were studied by Banaschewski in the category of archimedean $f$-rings (see [3, App. 2] and [4]). It is easy to see that the intersection of archimedean $\ell$-ideals is archimedean, and hence for each $S \subseteq A$ there is a least archimedean $\ell$-ideal containing $S$. As is standard, we call it the archimedean $\ell$-ideal generated by $S$.

As we pointed out in Section 2.2, for each $A \in \boldsymbol{b a} \ell$, the norm on $A$ is a weight function on $A$. Below we will work with a different weight function on $A$.

Definition 3.1. Let $A \in \boldsymbol{b a} \boldsymbol{\ell}$. Define $w_{A}$ on $A$ by $w_{A}(a)=\max \{\|a\|, 1\}$.

It is clear that $\left(A, w_{A}\right)$ is a weighted set. We use $w_{A}$ in order for a modal operator to be a weighted set morphism (see Lemma 4.2). The next definition is one of the main definitions of the paper and is motivated by the axioms defining a modal operator on $A \in \boldsymbol{b a} \boldsymbol{\ell}$.

Definition 3.2. Let $A \in \boldsymbol{b a \ell}$.

(1) Let $F(A)$ be the free object in $\boldsymbol{b a} \boldsymbol{\ell}$ on the weighted set $\left(A, w_{A}\right)$, and let $f_{A}: A \rightarrow F(A)$ be the associated map. We let $I_{A}$ be the archimedean $\ell$-ideal of $F(A)$ generated by the following elements, where $a, b \in A$ and $r \in \mathbb{R}$ :

(a) $f_{A}(a \wedge b)-f_{A}(a) \wedge f_{A}(b)$;

(b) $f_{A}(r)-r-(1-r) f_{A}(0)$;

(c) $f_{A}\left(a^{+}\right)-f_{A}(a)^{+}$;

(d) $f_{A}(a+r)-f_{A}(a)-f_{A}(r)+f_{A}(0)$;

(e) $f_{A}(r a)-f_{A}(r) f_{A}(a)$ if $0 \leq r$.

(2) Let $\mathscr{H}(A)=F(A) / I_{A}$ and $h_{A}: A \rightarrow \mathscr{H}(A)$ be the composition of $f_{A}$ with the quotient map $\pi: F(A) \rightarrow \mathscr{H}(A)$.

(3) For $a \in A$ let $\square_{a}=h_{A}(a)$.

Remark 3.3. The set $\left\{\square_{a} \mid a \in A\right\}$ generates $\mathscr{H}(A)$ (in $\boldsymbol{b} \boldsymbol{a} \boldsymbol{\ell}$ ), and these generators satisfy the following relations:

(F1) $\square_{a \wedge b}=\square_{a} \wedge \square_{b}$.

(F2) $\square_{r}=r+(1-r) \square_{0}$.

(F3) $\square_{a^{+}}=\left(\square_{a}\right)^{+}$.

(F4) $\square_{a+r}=\square_{a}+\square_{r}-\square_{0}$.

(F5) $\square_{r a}=\square_{r} \square_{a}$ if $0 \leq r$.

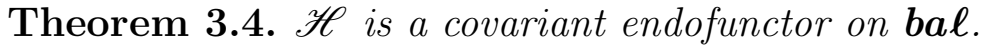

Proof. Let $\alpha: A \rightarrow B$ be a $\boldsymbol{b} \boldsymbol{a} \boldsymbol{\ell}$-morphism. Then $\alpha:\left(A, w_{A}\right) \rightarrow\left(B, w_{B}\right)$ is a weighted set morphism since

$$
w_{B}(\alpha(a))=\max \{\|\alpha(a)\|, 1\} \leq \max \{\|a\|, 1\}=w_{A}(a)
$$

for each $a \in A$. Therefore, there is a unique $\boldsymbol{b} \boldsymbol{a} \boldsymbol{\ell}$-morphism $\tau: F(A) \rightarrow F(B)$ making the following diagram commute.

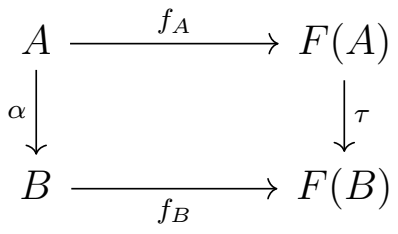


We show that $\tau\left(I_{A}\right) \subseteq I_{B}$. From this it will follow that there is an induced $\boldsymbol{b} \boldsymbol{a} \boldsymbol{\ell}$-morphism $\bar{\tau}: \mathscr{H}(A) \rightarrow \mathscr{H}(B)$ such that $\bar{\tau} \circ h_{A}=h_{B} \circ \alpha$. To see that $\tau\left(I_{A}\right) \subseteq I_{B}$, it suffices to show that the five sets of generators (a)-(e) of $I_{A}$ are sent to $I_{B}$ by $\tau$. Since the arguments are similar, we only give the argument for the generators of type (a).

Let $a, b \in A$. Then

$$
\begin{aligned}
\tau\left(f_{A}(a \wedge b)-f_{A}(a) \wedge f_{A}(b)\right) & =\tau f_{A}(a \wedge b)-\tau f_{A}(a) \wedge \tau f_{A}(b) \\
& =f_{B} \alpha(a \wedge b)-f_{B} \alpha(a) \wedge f_{B} \alpha(b) \\
& =f_{B}(\alpha(a) \wedge \alpha(b))-f_{B} \alpha(a) \wedge f_{B} \alpha(b) \\
& \in I_{B} .
\end{aligned}
$$

Therefore, $\tau$ induces a $\boldsymbol{b} \boldsymbol{a} \boldsymbol{\ell}$-morphism $\bar{\tau}: \mathscr{H}(A) \rightarrow \mathscr{H}(B)$. We set $\mathscr{H}(\alpha)=\bar{\tau}$. It follows that $\mathscr{H}(\alpha)$ is the unique $\boldsymbol{b} \boldsymbol{a} \boldsymbol{\ell}$-morphism that makes the following diagram commute.

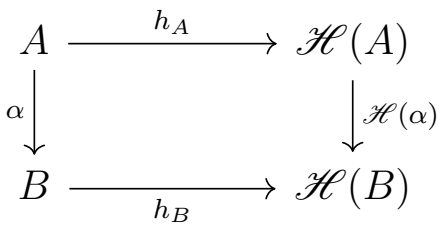

It is clear that $\mathscr{H}$ sends identity morphisms to identity morphisms. If $\alpha: A \rightarrow B$ and $\gamma: B \rightarrow C$ are $\boldsymbol{b a} \boldsymbol{\ell}$-morphisms, then

$$
\mathscr{H}(\gamma \circ \alpha) \circ h_{A}=h_{C} \circ \gamma \circ \alpha=\mathscr{H}(\gamma) \circ h_{B} \circ \alpha=\mathscr{H}(\gamma) \circ \mathscr{H}(\alpha) \circ h_{A} .
$$

Since $h_{A}[A]$ generates $\mathscr{H}(A)$, we see that $\mathscr{H}(\gamma \circ \alpha)=\mathscr{H}(\gamma) \circ \mathscr{H}(\alpha)$. Thus, $\mathscr{H}$ is a covariant functor.

Remark 3.5. From the commutativity $\mathscr{H}(\alpha) \circ h_{A}=h_{B} \circ \alpha$ it follows that $\mathscr{H}(\alpha)\left(\square_{a}\right)=\square_{\alpha(a)}$ for each $a \in A$. This will be used subsequently.

\section{4. $\operatorname{Alg}(\mathscr{H})$ AND $\boldsymbol{m b a} \boldsymbol{\ell}$}

In this section we show that the category $\operatorname{Alg}(\mathscr{H})$ of algebras for the endofunctor $\mathscr{H}$ is isomorphic to $\boldsymbol{m b a \ell}$. We start by recalling the definition of algebras for an endofunctor (see, e.g., [2, Def. 5.37]).

Definition 4.1. Let $\mathrm{C}$ be a category and $\mathscr{T}: \mathrm{C} \rightarrow \mathrm{C}$ an endofunctor on $\mathrm{C}$.

(1) An algebra for $\mathscr{T}$ is a pair $(A, f)$ where $A$ is an object of $\mathrm{C}$ and $f: \mathscr{T}(A) \rightarrow A$ is a C-morphism.

(2) Let $\left(A_{1}, f_{1}\right)$ and $\left(A_{2}, f_{2}\right)$ be two algebras for $\mathscr{T}$. A morphism between $\left(A_{1}, f_{1}\right)$ and $\left(A_{2}, f_{2}\right)$ is a C-morphism $\alpha: A_{1} \rightarrow A_{2}$ such that the following square is commutative.

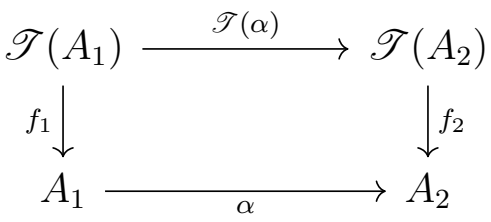


(3) Let $\operatorname{Alg}(\mathscr{T})$ be the category whose objects are algebras for $\mathscr{T}$ and whose morphisms are morphisms of algebras.

Lemma 4.2. If $(A, \square) \in \boldsymbol{m b a \ell}$, then $\square:\left(A, w_{A}\right) \rightarrow(A,\|\cdot\|)$ is a weighted set morphism.

Proof. Let $0 \leq r \in \mathbb{R}$. We first show that $\square r \leq \max \{r, 1\}$. If $r \leq 1$, then $\square r \leq \square 1=1$ by Remark 2.10. If $1 \leq r$, then $\square r=r+(1-r) \square 0 \leq r$ since $0 \leq \square 0$, again by Remark 2.10 , Therefore, $\square r \leq \max \{r, 1\}$.

We next show that $-\square r \leq \square(-r)$. We have $\square 0=\square(-r+r)=\square(-r)+\square r-\square 0$, so $0 \leq 2 \square 0=\square(-r)+\square r$. Thus, $-\square r \leq \square(-r)$.

To finish the proof, let $r=\|a\|$. Then $-r \leq a \leq r$, so $\square(-r) \leq \square a \leq \square r$. We have $\square r \leq \max \{r, 1\}$ and $-\square r \leq \square(-r)$. Therefore,

$$
-\max \{\|a\|, 1\}=-\max \{r, 1\} \leq-\square r \leq \square(-r) \leq \square a \leq \square r \leq \max \{r, 1\}=\max \{\|a\|, 1\},
$$

which implies that $\|\square a\| \leq \max \{\|a\|, 1\}=w_{A}(a)$. Thus, $\square:\left(A, w_{A}\right) \rightarrow(A,\|\cdot\|)$ is a weighted set morphism.

Lemma 4.3. There is a covariant functor $\mathscr{M}: \operatorname{Alg}(\mathscr{H}) \rightarrow \boldsymbol{m b a \ell}$ sending $(A, \sigma)$ to $\left(A, \square_{\sigma}\right)$, where $\square_{\sigma} a=\sigma\left(\square_{a}\right)$ for each $a \in A$, and an $\operatorname{Alg}(\mathscr{H})$-morphism $\alpha$ to itself.

Proof. Let $(A, \sigma) \in \operatorname{Alg}(\mathscr{H})$ and define $\square_{\sigma}$ on $A$ by $\square_{\sigma} a=\sigma\left(\square_{a}\right)$. It follows from Definition 2.9 and Remark 3.3 that $\left(A, \square_{\sigma}\right) \in \boldsymbol{m b a} \boldsymbol{\ell}$. If $\alpha:(A, \sigma) \rightarrow\left(A^{\prime}, \sigma^{\prime}\right)$ is an $\operatorname{Alg}(\mathscr{H})$ morphism,

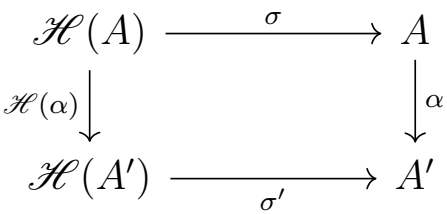

then

$$
\alpha\left(\square_{\sigma} a\right)=\alpha \sigma\left(\square_{a}\right)=\sigma^{\prime} \mathscr{H}(\alpha)\left(\square_{a}\right)=\sigma^{\prime}\left(\square_{\alpha(a)}\right)=\square_{\sigma^{\prime}} \alpha(a),
$$

where the second-to-last equality follows from Remark 3.5. Therefore, $\alpha$ is an $\boldsymbol{m b a} \boldsymbol{\ell}$ morphism. It is clear that $\mathscr{M}$ preserves identity morphisms and compositions. Thus, $\mathscr{M}$ is a covariant functor.

Lemma 4.4. There is a covariant functor $\mathscr{N}: \boldsymbol{m b a} \boldsymbol{l} \rightarrow \operatorname{Alg}(\mathscr{H}) \operatorname{sending}(A, \square)$ to $\left(A, \sigma_{\square}\right)$, where $\sigma_{\square}\left(\square_{a}\right)=\square$ a for each $a \in A$, and an mba $\boldsymbol{\ell}$-morphism $\alpha$ to itself.

Proof. Since $\square$ is a weighted set morphism by Lemma 4.2, there is a $\boldsymbol{b} \boldsymbol{a} \boldsymbol{\ell}$-morphism $\tau$ : $F(A) \rightarrow A$ satisfying $\tau f_{A}(a)=\square a$ by Theorem 2.8. It is clear from Definitions 2.9.(1) and $3.2(1)$ that $I_{A} \subseteq \operatorname{ker}(\tau)$, so there is a $\boldsymbol{b a} \boldsymbol{\ell}$-morphism $\sigma_{\square}: \mathscr{H}(A) \rightarrow A$ satisfying $\sigma_{\square}\left(\square_{a}\right)=$ $\square$ a. We set $\mathscr{N}(A, \square)=\left(A, \sigma_{\square}\right) \in \operatorname{Alg}(\mathscr{H})$. If $\alpha:(A, \square) \rightarrow\left(A^{\prime}, \square^{\prime}\right)$ is an $\boldsymbol{m b a} \boldsymbol{\ell}$-morphism, we show that $\alpha$ is an $\operatorname{Alg}(\mathscr{H})$-morphism. For this we show that the following diagram commutes.

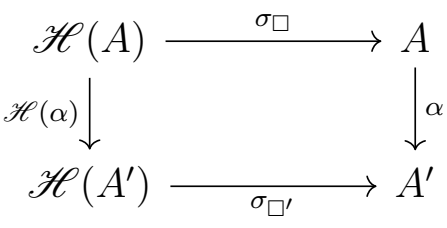


By Remark 3.5, $\mathscr{H}(\alpha)\left(\square_{a}\right)=\square_{\alpha(a)}$. Therefore, because $\alpha$ preserves $\square$, we have $\alpha \sigma_{\square}\left(\square_{a}\right)=$ $\alpha(\square a)=\square \alpha(a)$ and $\sigma_{\square^{\prime}} \mathscr{H}(\alpha)\left(\square_{a}\right)=\sigma_{\square^{\prime}}\left(\square_{\alpha(a)}\right)=\square \alpha(a)$. As $\left\{\square_{a} \mid a \in A\right\}$ generates $\mathscr{H}(A)$, we see that $\alpha \circ \sigma_{\square}=\sigma_{\square} \circ \mathscr{H}(\alpha)$, so $\alpha$ is an $\operatorname{Alg}(\mathscr{H})$-morphism. It is clear that $\mathscr{N}$ preserves identity morphisms and compositions. Thus, $\mathscr{N}$ is a covariant functor.

Theorem 4.5. The functors $\mathscr{M}$ and $\mathscr{N}$ yield an isomorphism of categories between $\operatorname{Alg}(\mathscr{H})$ and mbal.

Proof. Let $(A, \sigma) \in \operatorname{Alg}(\mathscr{H})$. Then $\mathscr{M}(A, \sigma)=\left(A, \square_{\sigma}\right)$. Therefore, $\mathscr{N} \mathscr{M}(A, \sigma)=\left(A, \sigma_{\square_{\sigma}}\right)$ where $\sigma_{\square_{\sigma}}\left(\square_{a}\right)=\square_{\sigma} a=\sigma\left(\square_{a}\right)$. Thus, $\sigma_{\square_{\sigma}}=\sigma$, and so $\mathscr{N} \mathscr{M}=1_{\mathrm{Alg}(\mathscr{H})}$.

Next, let $(A, \square) \in \boldsymbol{m b a} \boldsymbol{\ell}$. Then $\mathscr{N}(A, \square)=\left(A, \sigma_{\square}\right)$. Therefore, $\mathscr{M} \mathscr{N}(A, \square)=\left(A, \square_{\sigma_{\square}}\right)$. But $\square_{\sigma_{\square}} a=\sigma_{\square}\left(\square_{a}\right)=\square a$ by the definition of $\sigma_{\square}$, so $\square_{\sigma_{\square}}=\square$. Thus, $\mathscr{M} \mathscr{N}=1_{\text {mbal }}$. Consequently, $\mathscr{M}$ and $\mathscr{N}$ yield an isomorphism between $\operatorname{Alg}(\mathscr{H})$ and $\boldsymbol{m b a} \boldsymbol{\ell}$.

\section{5. $\mathscr{H}$ And the Vietoris endofunctor}

In this section we relate $\mathscr{H}$ to the Vietoris endofunctor $\mathscr{V}:$ KHaus $\rightarrow$ KHaus by showing that the Yosida space $Y_{\mathscr{H}(A)}$ for $A \in \boldsymbol{b a} \boldsymbol{\ell}$ is homeomorphic to $\mathscr{V}\left(Y_{A}\right)$.

Let $X \in$ KHaus. We recall that the Vietoris space $\mathscr{V}(X)$ is the set of closed subsets of $X$, topologized as follows. If $U$ is an open subset of $X$, let

$$
\begin{aligned}
\square_{U} & =\{F \in \mathscr{V}(X) \mid F \subseteq U\}, \\
\triangleright_{U} & =\{F \in \mathscr{V}(X) \mid F \cap U \neq \varnothing\} .
\end{aligned}
$$

The Vietoris topology on $\mathscr{V}(X)$ is the topology with the subbasis

$$
\left\{\square_{U} \cap \diamond_{V} \mid U, V \text { open in } X\right\} .
$$

We extend $\mathscr{V}$ to a functor as follows. If $\varphi: X \rightarrow Y$ is a continuous function between compact Hausdorff spaces, define $\mathscr{V}(\varphi): \mathscr{V}(X) \rightarrow \mathscr{V}(Y)$ by $\mathscr{V}(\varphi)(F)=\varphi(F)$, the image of $F$ under $\varphi$. It is well known that $\mathscr{V}(\varphi)$ is a well-defined continuous map.

Remark 5.1. The Vietoris space of $X$ is usually defined as the space of nonempty closed subsets of $X$ (see, e.g., [12, p. 120]). However, we follow [17, p. 111] in including $\varnothing$ in $\mathscr{V}(X)$. This is necessary for our considerations since the continuous relation $R$ on $X$ may not be serial, and hence there may be $x \in X$ with $R[x]=\varnothing$. Therefore, $\rho_{R}(x)=\varnothing$, and we need $\varnothing \in \mathscr{V}(X)$ for $\rho_{R}$ to be well defined. However, in Section 8 we will consider $\mathscr{V}^{*}(X)=\mathscr{V}(X) \backslash\{\varnothing\}$ and relate it to the full subcategory of $\boldsymbol{m b a} \boldsymbol{\ell}$ corresponding to those $(X, R)$ where $R$ is a serial relation. This subcategory will be characterized by the identity $\square 0=0$.

Lemma 5.2. Let $A \in \boldsymbol{b a} \boldsymbol{\ell}$. Define $g_{A}: A \rightarrow C\left(\mathscr{V} Y_{A}\right)$ by

$$
g_{A}(a)(F)= \begin{cases}\inf \zeta_{A}(a)(F) & \text { if } F \neq \varnothing ; \\ 1 & \text { if } F=\varnothing .\end{cases}
$$

Then $g_{A}:\left(A, w_{A}\right) \rightarrow\left(C\left(\mathscr{V} Y_{A}\right),\|\cdot\|\right)$ is a well-defined weighted set morphism. 
Proof. To simplify notation we write $g$ for $g_{A}$. To see that $g$ is well defined it is sufficient to show that $g(a)$ is continuous for each $a \in A$. Let $r, s \in \mathbb{R}$ with $r<s$. We show that

$$
g(a)^{-1}(r, s)= \begin{cases}\square_{\zeta_{A}(a)^{-1}(r, \infty)} \cap \diamond_{\zeta_{A}(a)^{-1}(-\infty, s)} & \text { if } 1 \notin(r, s) \\ \left(\square_{\zeta_{A}(a)^{-1}(r, \infty)} \cap \diamond_{\zeta_{A}(a)^{-1}(-\infty, s)} \cup \square_{\varnothing}\right. & \text { if } 1 \in(r, s) .\end{cases}
$$

Suppose that $1 \notin(r, s)$. Then $g(a)(F) \in(r, s)$ implies that $F \neq \varnothing$. Therefore, since $F$ is compact and hence $\zeta_{A}(a)$ attains its infimum on $F$, we have

$$
\begin{aligned}
F \in g(a)^{-1}(r, s) \quad \text { iff } & r<\inf \zeta_{A}(a)(F)<s \\
& \text { iff } \quad r<\min \zeta_{A}(a)(F)<s \\
& \text { iff } \quad F \in \square_{\zeta_{A}(a)^{-1}(r, \infty)} \quad \diamond_{\zeta_{A}(a)^{-1}(-\infty, s)} .
\end{aligned}
$$

On the other hand, if $1 \in(r, s)$, then $\varnothing \in g(a)^{-1}(r, s)$. Therefore, since $\square_{\varnothing}=\{\varnothing\}$, the calculation above yields the second case. Thus, $g(a)$ is continuous.

It is left to show that $g$ is a weighted set morphism. Let $a \in A$. Then $w_{A}(a)=\max \{\|a\|, 1\}$. Suppose that $\|a\|=r$. Then $-r \leq a \leq r$. If $F$ is nonempty, then $-r \leq \inf \zeta_{A}(a)(F) \leq r$, so $\left|\inf \zeta_{A}(a)(F)\right| \leq r$. Also, $g(a)(\varnothing)=1$. Therefore,

$$
\begin{aligned}
\|g(a)\| & =\sup \left\{|g(a)(F)| \mid F \in \mathscr{V}\left(Y_{A}\right)\right\}=\sup \left\{\left\{\left|\inf \zeta_{A}(a)(F)\right| \mid F \neq \varnothing\right\} \cup\{1\}\right\} \\
& =\max \left\{\sup \left\{\left|\inf \zeta_{A}(a)(F)\right| \mid F \neq \varnothing\right\}, 1\right\} \leq \max \{r, 1\}=w_{A}(a) .
\end{aligned}
$$

Thus, $g:\left(A, w_{A}\right) \rightarrow\left(C\left(\mathscr{V} Y_{A}\right),\|\cdot\|\right)$ is a weighted set morphism.

Remark 5.3. In the proof of Lemma 5.4 we identify $X$ with $Y_{C(X)}$ via the homeomorphism $\varepsilon_{X}: X \rightarrow Y_{C(X)}$ given in Section 2.1. We also identify $Y_{A}$ with $\operatorname{hom}_{b a \ell}(A, \mathbb{R})$ as follows. If $M \in Y_{A}$, then it is well known that $A / M \cong \mathbb{R}$ (see, e.g., [16, Cor. 2.7]), so there is a ba $\boldsymbol{\ell}$-morphism $A \rightarrow \mathbb{R}$ sending $a \in A$ to $r \in \mathbb{R}$ iff $a-r \in M$. Conversely, $\rho \in \operatorname{hom}_{\text {ba } \boldsymbol{\ell}}(A, \mathbb{R})$ goes to $\operatorname{ker}(\rho) \in Y_{A}$.

Lemma 5.4. There is a (unique) ba $\ell$-morphism $\tau_{A}: F(A) \rightarrow C\left(\mathscr{V} Y_{A}\right)$ satisfying $\tau_{A} \circ f_{A}=$ $g_{A}$, the image of $\tau_{A}$ is uniformly dense in $C\left(\mathscr{V} Y_{A}\right)$, and $\operatorname{ker}\left(\tau_{A}\right)$ contains $I_{A}$. Therefore, there is a (unique) bal-morphism $\eta_{A}: \mathscr{H}(A) \rightarrow C\left(\mathscr{V} Y_{A}\right)$ satisfying $\eta_{A} \circ h_{A}=g_{A}$ and whose image is uniformly dense in $C\left(\mathscr{V} Y_{A}\right)$.

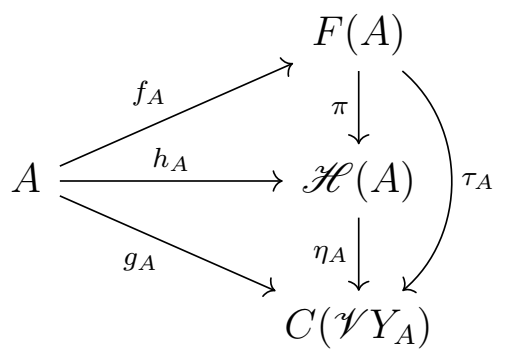

Proof. The existence and uniqueness of $\tau_{A}$ follows from Lemma 5.2 and Theorem 2.8 , To show that the image of $\tau_{A}$ is uniformly dense, by Lemma 2.6 (2) it suffices to show that $\mathscr{Y}\left(\tau_{A}\right)$ : $Y_{C\left(\mathscr{V} Y_{A}\right)} \rightarrow Y_{F(A)}$ is 1-1. We may identify $Y_{F(A)}$ with $\operatorname{hom}_{\boldsymbol{b a \ell}}(F(A), \mathbb{R})$ and $Y_{C\left(\mathscr{V} Y_{A}\right)}$ with 
$\mathscr{V}\left(Y_{A}\right)$ by Remark 5.3. Under these identifications, if $F \in \mathscr{V} Y_{A}$ we let $\rho_{F} \in \operatorname{hom}_{\text {ba } \ell}(F(A), \mathbb{R})$ be the corresponding homomorphism. For $a \in A$ and $r \in \mathbb{R}$ we have

$$
\begin{aligned}
& \rho_{F}\left(f_{A}(a)\right)=r \quad \text { iff } f_{A}(a)-r \in \mathscr{Y}\left(\tau_{A}\right)\left(\varepsilon_{\mathscr{V} Y_{A}}(F)\right) \\
& \text { iff } f_{A}(a)-r \in \tau_{A}^{-1}\left(\varepsilon_{\mathscr{V} Y_{A}}(F)\right) \\
& \text { iff } \tau_{A} f_{A}(a)-r \in \varepsilon_{\mathscr{V} Y_{A}}(F) \\
& \text { iff } \tau_{A} f_{A}(a)(F)=r \\
& \text { iff } g_{A}(a)(F)=r .
\end{aligned}
$$

Therefore, $\rho_{F}$ satisfies $\rho_{F}\left(f_{A}(a)\right)=\inf \zeta_{A}(a)(F)$ if $F \neq \varnothing$, and $\rho_{\varnothing}$ is the function sending each $f_{A}(a)$ to 1 . To see that $\mathscr{Y}\left(\tau_{A}\right)$ is $1-1$, suppose that $C \neq D$. If one of $C, D$ is empty, say $C=\varnothing$, then $\rho_{C} f_{A}(0)=1$ and $\rho_{D} f_{A}(0)=\inf \zeta_{A}(0)(D)=0$ since $D$ is nonempty. Therefore, $\rho_{C} \neq \rho_{D}$. If $C, D \neq \varnothing$, without loss of generality we may assume that $C \nsubseteq D$. Then there is $y \in Y_{A}$ with $y \in C$ and $y \notin D$. Since $Y_{A}$ is compact Hausdorff, there is $b \in C\left(Y_{A}\right)$ with $0 \leq b \leq 1, b(D)=\{1\}$ and $b(y)=0$. Because $\zeta_{A}[A]$ is uniformly dense in $C\left(Y_{A}\right)$, there is $a \in A$ with $\left\|b-\zeta_{A}(a)\right\|<1 / 3$. Therefore, $\inf \zeta_{A}(a)(D) \geq 2 / 3$ and $\inf \zeta_{A}(a)(C) \leq 1 / 3$. This shows that $\rho_{C} f_{A}(a) \neq \rho_{D} f_{A}(a)$, so $\rho_{C} \neq \rho_{D}$. Thus, $\mathscr{Y}\left(\tau_{A}\right)$ is $1-1$, and hence the image of $\tau_{A}: F(A) \rightarrow C\left(\mathscr{V} Y_{A}\right)$ is uniformly dense.

To show that $I_{A} \subseteq \operatorname{ker}\left(\tau_{A}\right)$, it is sufficient to show that $\operatorname{ker}\left(\tau_{A}\right)$ contains all five classes of generators of $I_{A}$. Because the proof is similar to that of [7, Lem. 3.8], we only demonstrate (a).

Let $a, b \in A$. We have

$\tau_{A}\left(f_{A}(a \wedge b)-f_{A}(a) \wedge f_{A}(b)\right)=\tau_{A} f_{A}(a \wedge b)-\tau_{A} f_{A}(a) \wedge \tau_{A} f_{A}(b)=g_{A}(a \wedge b)-g_{A}(a) \wedge g_{A}(b)$.

Therefore, we need to prove that $g_{A}(a \wedge b)=g_{A}(a) \wedge g_{A}(b)$. Both sides send $\varnothing$ to 1 . Suppose that $F \in \mathscr{V}\left(Y_{A}\right)$ is nonempty. Then

$$
\begin{aligned}
g_{A}(a \wedge b)(F) & =\inf \left(\zeta_{A}(a) \wedge \zeta_{A}(b)\right)(F)=\min \left(\zeta_{A}(a) \wedge \zeta_{A}(b)\right)(F) \\
& =\min \left\{\left(\zeta_{A}(a) \wedge \zeta_{A}(b)\right)(x) \mid x \in F\right\} \\
& =\min \left\{\min \left\{\zeta_{A}(a)(x), \zeta_{A}(b)(x)\right\} \mid x \in F\right\} \\
& =\min \left\{\min \zeta_{A}(a)(F), \min \zeta_{A}(b)(F)\right\} \\
& =\left(g_{A}(a) \wedge g_{A}(b)\right)(F) .
\end{aligned}
$$

Thus, $g_{A}(a \wedge b)=g_{A}(a) \wedge g_{A}(b)$.

We next show that $\eta_{A}$ is $1-1$. For this we require a technical result, which is an analogue of [7, Prop. 4.8].

Definition 5.5. Let $A \in \boldsymbol{b a} \boldsymbol{\ell}$.

(1) If $x \in Y_{\mathscr{H}(A)}$, set $\square^{-1} x=\left\{a \in A \mid \square_{a} \in x\right\}$.

(2) If $S \subseteq A$, set $S^{+}=\{s \in S \mid 0 \leq s\}$.

(3) Define a binary relation $R^{\square} \subseteq Y_{\mathscr{H}(A)} \times Y_{A}$ by setting $x R^{\square} y$ if $y^{+} \subseteq \square^{-1} x$ for each $x \in Y_{\mathscr{H}(A)}$ and $y \in Y_{A}$.

Proposition 5.6. Let $A \in$ ba $\ell$ and $x \in Y_{\mathscr{H}(A)}$. Then $\left(\square^{-1} x\right)^{+}=\bigcup\left\{y^{+} \mid y \in Y_{A}, x R^{\square} y\right\}$. 
Proof. The proof is the same as that of [7, Prop. 4.8] after replacing $\square a$ with $\square_{a}$ and $R_{\square}$ with $R^{\square}$.

Lemma 5.7. Let $\rho: \mathscr{H}(A) \rightarrow \mathbb{R}$ be a ba $\mathbf{\ell}$-morphism.

(1) $\rho\left(\square_{0}\right) \in\{0,1\}$.

(2) If $\rho\left(\square_{0}\right)=1$, then $\rho\left(\square_{a}\right)=1$ for each $a \in A$.

Proof. (1) If we set $r=0=a$ in (F5), we get $\square_{0} \square_{0}=\square_{0}$, so $\square_{0}$ is an idempotent. Therefore, $\rho\left(\square_{0}\right) \in \mathbb{R}$ is an idempotent, and hence $\rho\left(\square_{0}\right) \in\{0,1\}$.

(2) Suppose that $\rho\left(\square_{0}\right)=1$. By (F5), $\square_{0} \square_{a}=\square_{0}$ for each $a \in A$. So applying $\rho$ to both sides yields $\rho\left(\square_{a}\right)=1$.

Theorem 5.8. For $A \in \mathbf{b a \ell}$, the Yosida space of $\mathscr{H}(A)$ is homeomorphic to $\mathscr{V}\left(Y_{A}\right)$.

Proof. The map $\eta_{A}: \mathscr{H}(A) \rightarrow C\left(\mathscr{V} Y_{A}\right)$ induces a continuous map $\mathscr{Y}\left(\eta_{A}\right): Y_{C\left(\mathscr{V} Y_{A}\right)} \rightarrow Y_{\mathscr{H}(A)}$. We identify $Y_{C\left(\mathscr{Y} Y_{A}\right)}$ with $\mathscr{V}\left(Y_{A}\right)$ and $Y_{\mathscr{H}(A)}$ with $\operatorname{hom}_{b a \ell}(\mathscr{H}(A), \mathbb{R})$ as in Remark 5.3, As we saw in the proof of Lemma [5.4, under these identifications $\mathscr{Y}\left(\eta_{A}\right)(F):=\rho_{F}$ satisfies $\rho_{F}\left(\square_{a}\right)=\inf \zeta_{A}(a)(F)$ if $F$ is nonempty, and $\rho_{F}\left(\square_{a}\right)=1$ if $F=\varnothing$. By Lemma [5.4, the image of $\eta_{A}$ is uniformly dense in $C\left(\mathscr{V} Y_{A}\right)$. Therefore, $\mathscr{Y}\left(\eta_{A}\right)$ is 1-1 by Lemma 2.6(2).

To show that $\mathscr{Y}\left(\eta_{A}\right)$ is onto, let $\rho: \mathscr{H}(A) \rightarrow \mathbb{R}$ be a $\boldsymbol{b} \boldsymbol{a} \boldsymbol{\ell}$-morphism. If $\rho\left(\square_{0}\right)=1$, then $\rho\left(\square_{a}\right)=1$ for all $a \in A$ by Lemma $5.7(2)$. Therefore, $\rho$ and $\rho_{\varnothing}$ agree on each $\square_{a}$. Since these generate $\mathscr{H}(A)$, we see that $\rho=\rho_{\varnothing}$. By Lemma $5.7(1)$, we now may assume that $\rho\left(\square_{0}\right)=0$. By (F2), $\rho\left(\square_{r}\right)=r$ for each $r \in \mathbb{R}$. Let

$$
S=\left\{\left(a-\rho\left(\square_{a}\right)\right)^{-} \mid a \in A\right\}
$$

and $F=\left\{M \in Y_{A} \mid S \subseteq M\right\}$, a closed subset of $Y_{A}$. We claim that $\rho=\rho_{F}$. Let $a \in A$ and $y \in F$. Then $\left(a-\rho\left(\square_{a}\right)\right)^{-} \in y$. This means $0 \leq\left(\zeta_{A}(a)-\rho\left(\square_{a}\right)\right)(y)$ by [9, Rem. 2.11], so $\rho\left(\square_{a}\right) \leq \zeta_{A}(a)(y)$. Since this is true for all $y \in F$, we see that $\rho\left(\square_{a}\right) \leq \inf \zeta_{A}(a)(F)$. Thus, it suffices to prove that for each $a \in A$ there is $y \in F$ with $\zeta_{A}(a)(y)=\rho\left(\square_{a}\right)$. In other words, we need to show that there is $y \in F$ with $a-\rho\left(\square_{a}\right) \in y$.

Let $x=\operatorname{ker}(\rho) \in Y_{\mathscr{H}(A)}$. If $a \in A$, then

$$
\rho\left(\square_{a-\rho\left(\square_{a}\right)}\right)=\rho\left(\square_{a}+\square_{-\rho\left(\square_{a}\right)}-\square_{0}\right)=\rho\left(\square_{a}\right)-\rho\left(\square_{a}\right)=0
$$

by (F4) and the fact that $\rho\left(\square_{r}\right)=r$. From this and (F3) we see that

$$
\rho\left(\square_{\left(a-\rho\left(\square_{a}\right)\right)^{+}}\right)=\rho\left(\square_{a-\rho\left(\square_{a}\right)}^{+}\right)=\rho\left(\square_{a-\rho\left(\square_{a}\right)}\right)^{+}=\max \left\{\rho\left(\square_{a-\rho\left(\square_{a}\right)}\right), 0\right\}=\max \{0,0\}=0,
$$

which implies that $\left(a-\rho\left(\square_{a}\right)\right)^{+} \in \square^{-1} x$. By Proposition 5.6, there is $y \in Y_{A}$ with $x R^{\square} y$ and $\left(a-\rho\left(\square_{a}\right)\right)^{+} \in y$. We show that these two facts imply that $y \in F$ and $\rho\left(\square_{a}\right)=\zeta_{A}(a)(y)$. Let $b \in A$. Since $A / y \cong \mathbb{R}$, there is $r \in \mathbb{R}$ with $b-r \in y$. Therefore, $(b-r)^{+} \in y$, so $\square_{(b-r)^{+}} \in x$. Because $x=\operatorname{ker}(\rho)$,

$$
0=\rho\left(\square_{(b-r)^{+}}\right)=\rho\left(\square_{b-r}^{+}\right)=\rho\left(\square_{b-r}\right)^{+}=\max \left\{\rho\left(\square_{b-r}\right), 0\right\}=\max \left\{\rho\left(\square_{b}\right)-r, 0\right\},
$$

so $\rho\left(\square_{b}\right) \leq r$. Consequently, $b+y=r+y \geq \rho\left(\square_{b}\right)+y$, and hence $b-\rho\left(\square_{b}\right)+y \geq 0+y$. This implies that $\left(b-\rho\left(\square_{b}\right)\right)^{-} \in y$. Since this is true for all $b \in A$, we get $S \subseteq y$, so $y \in F$. Moreover, for $b=a$ we have $\left(a-\rho\left(\square_{a}\right)\right)^{+},\left(a-\rho\left(\square_{a}\right)\right)^{-} \in y$, so $a-\rho\left(\square_{a}\right) \in y$. By the above, this shows that $\rho=\rho_{F}$, so $\mathscr{Y}\left(\eta_{A}\right)$ is onto. Thus, $\mathscr{Y}\left(\eta_{A}\right)$ is a homeomorphism. 
Remark 5.9. By Theorem 5.8, $Y_{\mathscr{H}(A)}$ is homeomorphic to $\mathscr{V}\left(Y_{A}\right)$. Under this homeomorphism, $R^{\square} \subseteq Y_{\mathscr{H}(A)} \times Y_{A}$ is identified with the relation $R \subseteq \mathscr{V}\left(Y_{A}\right) \times Y_{A}$ given by $F R y$ iff $y \in F$. From this it follows that $R[F]=F$, and for $U \subseteq Y_{A}$ open, we have $R^{-1}[U]=\diamond_{U}$ and $R^{-1}\left[Y_{A} \backslash U\right]=\mathscr{V}\left(Y_{A}\right) \backslash \square_{U}$. Consequently, $R$ is a continuous relation, and hence so is $R^{\square}$.

\section{6. $\operatorname{Alg}(\mathscr{H})$ AND $\operatorname{Coalg}(\mathscr{V})$}

In this section we lift the dual adjunction between $\boldsymbol{b a} \boldsymbol{\ell}$ and KHaus to a dual adjunction between $\operatorname{Alg}(\mathscr{H})$ and Coalg $(\mathscr{V})$. We show that this dual adjunction restricts to a dual equivalence between the reflective subcategory $\operatorname{Alg}^{u}(\mathscr{H})$ of $\operatorname{Alg}(\mathscr{H})$ and $\operatorname{Coalg}(\mathscr{V})$. The category $\operatorname{Alg}^{u}(\mathscr{H})$ consists of those $(A, \alpha) \in \operatorname{Alg}(\mathscr{H})$ where $A \in \boldsymbol{u} \boldsymbol{b a} \boldsymbol{\ell}$. This dual equivalence lifts Gelfand duality. We conclude the section by giving an alternate description of $\operatorname{Alg}^{u}(\mathscr{H})$ as $\operatorname{Alg}\left(\mathscr{H}^{u}\right)$ where $\mathscr{H}^{u}$ is the endofunctor $\mathscr{C} \mathscr{Y} \mathscr{H}: \boldsymbol{u b a} \boldsymbol{l} \rightarrow \boldsymbol{u b a} \boldsymbol{\ell}$.

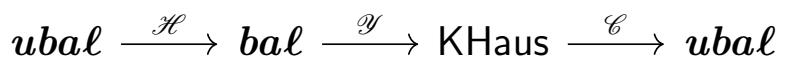

We start by recalling the definition of coalgebras (see, e.g., [22, Def. 9.1]), which is dual to the definition of algebras for an endofunctor.

\section{Definition 6.1.}

(1) A coalgebra for an endofunctor $\mathscr{T}: \mathrm{C} \rightarrow \mathrm{C}$ is a pair $(B, g)$ where $B$ is an object of $\mathrm{C}$ and $g: B \rightarrow \mathscr{T}(B)$ is a C-morphism.

(2) A morphism between two coalgebras $\left(B_{1}, g_{1}\right)$ and $\left(B_{2}, g_{2}\right)$ for $\mathscr{T}$ is a C-morphism $\alpha: B_{1} \rightarrow B_{2}$ such that the following square is commutative.

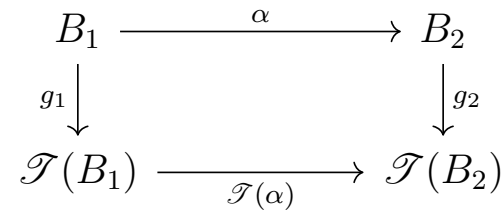

(3) Let Coalg $(\mathscr{T})$ be the category whose objects are coalgebras for $\mathscr{T}$ and whose morphisms are morphisms of coalgebras.

Lemma 6.2. Let $\gamma: A \rightarrow A^{\prime}$ be a ba $\boldsymbol{\ell}$-morphism. Then the following diagram is commutative.

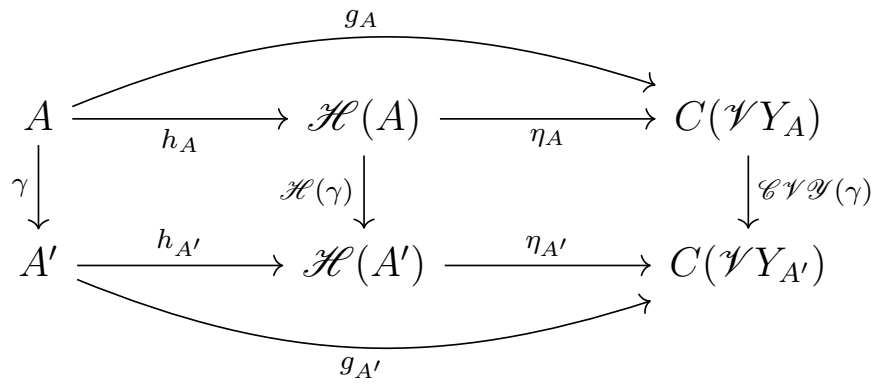

Proof. By Remark 3.5, $\mathscr{H}(\gamma)\left(h_{A}(a)\right)=\mathscr{H}(\gamma)\left(\square_{a}\right)=\square_{\gamma(a)}=h_{A^{\prime}} \gamma(a)$ for each $a \in A$. This shows that the left square of the diagram is commutative. By definition, $g_{A}=\eta_{A} \circ h_{A}$ and 
$g_{A^{\prime}}=\eta_{A^{\prime}} \circ h_{A^{\prime}}$. We next show that the outside square is commutative, from which we then derive that the right square is commutative. Let $a \in A$ and $F \in \mathscr{V}\left(Y_{A^{\prime}}\right)$. If $F=\varnothing$, then

$$
\mathscr{C} \mathscr{V} \mathscr{Y}(\gamma)\left(g_{A}(a)\right)(\varnothing)=g_{A}(a)(\mathscr{Y}(\gamma)(\varnothing))=g_{A}(a)(\varnothing)=1=g_{A^{\prime}} \gamma(a)(\varnothing)
$$

If $F \neq \varnothing$, then naturality of $\zeta$ yields

$$
\begin{aligned}
\mathscr{C} \mathscr{V} \mathscr{Y}(\gamma)\left(g_{A}(a)\right)(F) & =g_{A}(a)(\mathscr{Y}(\gamma)(F))=\inf \left(\zeta_{A}(a) \mathscr{Y}(\gamma)\right)(F) \\
& =\inf \left(\mathscr{C} \mathscr{Y}(\gamma) \circ \zeta_{A}\right)(a)(F)=\inf \zeta_{A^{\prime}}(\gamma(a))(F) \\
& =g_{A^{\prime}} \gamma(a)(F) .
\end{aligned}
$$

Thus, $\mathscr{C} \mathscr{V} \mathscr{Y}(\gamma) \circ g_{A}=g_{A^{\prime}} \circ \gamma$. Finally, to see that the right square is commutative,

$$
\mathscr{C} \mathscr{V} \mathscr{Y}(\gamma) \circ \eta_{A} \circ h_{A}=\mathscr{C} \mathscr{V} \mathscr{Y}(\gamma) \circ g_{A}=g_{A^{\prime}} \circ \gamma=\eta_{A^{\prime}} \circ \mathscr{H}(\gamma) \circ h_{A}
$$

This yields $\mathscr{C} \mathscr{V} \mathscr{Y}(\gamma) \circ \eta_{A}=\eta_{A^{\prime}} \circ \mathscr{H}(\gamma)$ because the image of $h_{A}$ generates $\mathscr{H}(A)$.

Proposition 6.3. There is a contravariant functor $\mathscr{F}: \operatorname{Alg}(\mathscr{H}) \rightarrow \operatorname{Coalg}(\mathscr{V})$.

Proof. By the proof of Theorem [5.8, if $A \in \boldsymbol{b a} \boldsymbol{\ell}$, then $\mathscr{Y}\left(\eta_{A}\right)$ is a homeomorphism. For $(A, \alpha) \in \operatorname{Alg}(\mathscr{H})$, we set $\mathscr{F}(A, \alpha)=\left(Y_{A}, \mathscr{F}_{\alpha}\right) \in \operatorname{Coalg}(\mathscr{V})$, where

$$
\begin{gathered}
\mathscr{F}_{\alpha}=\varepsilon_{\mathscr{V}\left(Y_{A}\right)}^{-1} \circ \mathscr{Y}\left(\eta_{A}\right)^{-1} \circ \mathscr{Y}(\alpha): Y_{A} \rightarrow \mathscr{V}\left(Y_{A}\right), \\
Y_{A} \stackrel{\mathscr{Y}(\alpha)}{\longrightarrow} Y_{\mathscr{H}(A)} \stackrel{\mathscr{Y}\left(\eta_{A}\right)^{-1}}{\longrightarrow} Y_{C\left(\mathscr{V} Y_{A}\right)} \stackrel{\varepsilon_{\mathscr{V}\left(Y_{A}\right)}^{-1}}{\longrightarrow} \mathscr{V}\left(Y_{A}\right) \\
\mathscr{F}_{\alpha}
\end{gathered}
$$

If $\gamma:(A, \alpha) \rightarrow\left(A^{\prime}, \alpha^{\prime}\right)$ is an $\operatorname{Alg}(\mathscr{H})$-morphism

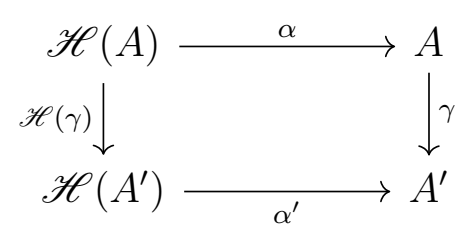

then $\mathscr{Y}(\gamma): Y_{A^{\prime}} \rightarrow Y_{A}$ is a continuous map. We define $\mathscr{F}(\gamma)=\mathscr{Y}(\gamma)$. To see that $\mathscr{Y}(\gamma)$ is a Coalg $(\mathscr{V})$-morphism, we show that the following diagram is commutative.

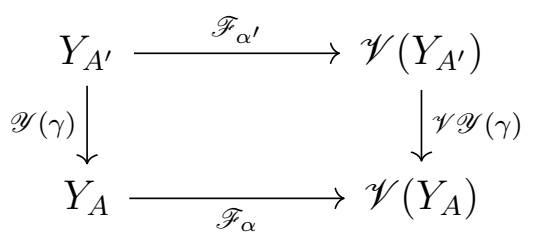

To see this we first show that the following diagram is commutative.

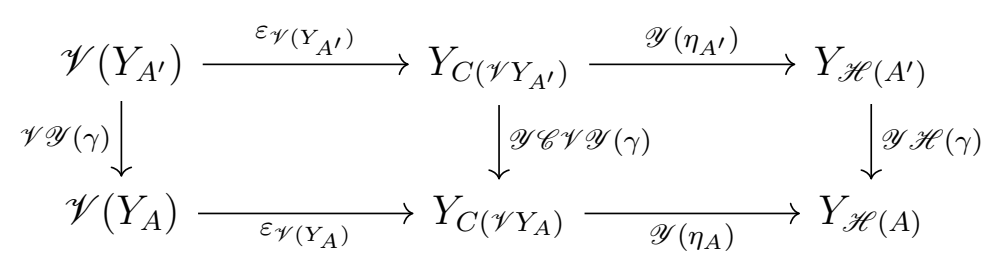


The left square commutes due to the naturality of $\varepsilon$. For the right square, $\mathscr{Y} \mathscr{H}(\gamma) \circ \mathscr{Y}\left(\eta_{A^{\prime}}\right)=$ $\mathscr{Y}\left(\eta_{A^{\prime}} \circ \mathscr{H}(\gamma)\right)$ and $\mathscr{Y}\left(\eta_{A}\right) \circ \mathscr{Y} \mathscr{C} \mathscr{V} \mathscr{Y}(\gamma)=\mathscr{Y}\left(\mathscr{C} \mathscr{V} \mathscr{Y}(\gamma) \circ \eta_{A}\right)$. These are equal by Lemma 6.2, Now, we show that Diagram (1) commutes. The equation

$$
\mathscr{V} \mathscr{Y}(\gamma) \circ \mathscr{F}_{\alpha^{\prime}}=\mathscr{F}_{\alpha} \circ \mathscr{Y}(\gamma)
$$

is equivalent to

$$
\mathscr{V} \mathscr{Y}(\gamma) \circ \varepsilon_{\mathscr{V}\left(Y_{A^{\prime}}\right)}^{-1} \circ \mathscr{Y}\left(\eta_{A^{\prime}}\right)^{-1} \circ \mathscr{Y}\left(\alpha^{\prime}\right)=\varepsilon_{\mathscr{V}\left(Y_{A}\right)}^{-1} \circ \mathscr{Y}\left(\eta_{A}\right)^{-1} \circ \mathscr{Y}(\alpha) \circ \mathscr{Y}(\gamma)
$$

and therefore is equivalent to

$$
\mathscr{Y}\left(\eta_{A}\right) \circ \varepsilon_{\mathscr{V}\left(Y_{A}\right)} \circ \mathscr{V} \mathscr{Y}(\gamma) \circ \varepsilon_{\mathscr{V}\left(Y_{A^{\prime}}\right)}^{-1} \circ \mathscr{Y}\left(\eta_{A^{\prime}}\right)^{-1} \circ \mathscr{Y}\left(\alpha^{\prime}\right)=\mathscr{Y}(\alpha) \circ \mathscr{Y}(\gamma)
$$

Using the commutativity of Diagram (2) and Equation (3), we see that commutativity of Diagram (11) is equivalent to the equation

$$
\mathscr{Y} \mathscr{H}(\gamma) \circ \mathscr{Y}\left(\alpha^{\prime}\right)=\mathscr{Y}(\alpha) \circ \mathscr{Y}(\gamma)
$$

Since $\gamma$ is an $\operatorname{Alg}(\mathscr{H})$-morphism, we have $\gamma \circ \alpha=\alpha^{\prime} \circ \mathscr{H}(\gamma)$. Applying $\mathscr{Y}$ to both sides then yields the commutativity of Diagram (11). Therefore, $\mathscr{Y}(\gamma)$ is a $\operatorname{Coalg}(\mathscr{V})$-morphism. It is then straightforward to see that $\mathscr{F}$ is a contravariant functor.

Proposition 6.4. There is a contravariant functor $\mathscr{G}: \operatorname{Coalg}(\mathscr{V}) \rightarrow \operatorname{Alg}(\mathscr{H})$.

Proof. Let $(X, \sigma) \in \operatorname{Coalg}(\mathscr{V})$. Then $\mathscr{C}(\sigma): C(\mathscr{V} X) \rightarrow C(X)$ is a $\boldsymbol{b} \boldsymbol{a} \boldsymbol{\ell}$-morphism. We set $\mathscr{G}(X, \sigma)=\left(X, \mathscr{G}_{\sigma}\right)$, where $\mathscr{G}_{\sigma}=\mathscr{C}(\sigma) \circ \mathscr{C} \mathscr{V}\left(\varepsilon_{X}\right) \circ \eta_{C(X)}$.

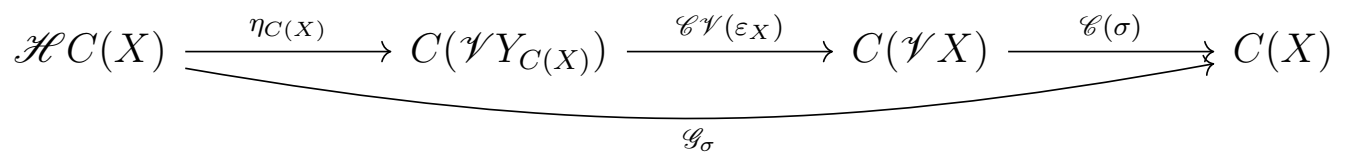

If $\varphi:(X, \sigma) \rightarrow\left(X^{\prime}, \sigma^{\prime}\right)$ is a Coalg $(\mathscr{V})$-morphism,

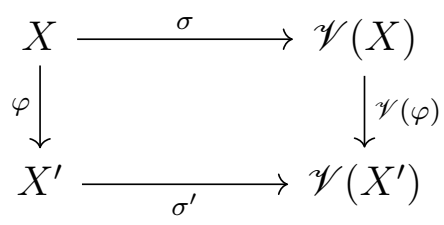

We define $\mathscr{G}(\varphi)=\mathscr{C}(\varphi)$. We need to show that $\mathscr{C}(\varphi)$ is an $\operatorname{Alg}(\mathscr{H})$-morphism.

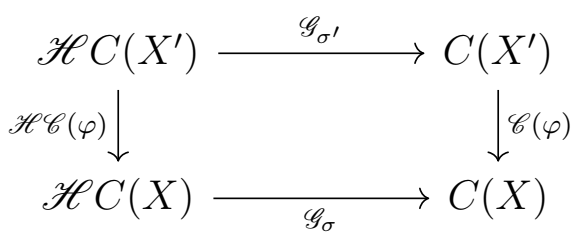


We have

$$
\begin{aligned}
\mathscr{C}(\varphi) \circ \mathscr{G}_{\sigma^{\prime}} & =\mathscr{C}(\varphi) \circ \mathscr{C}\left(\sigma^{\prime}\right) \circ \mathscr{C} \mathscr{V}\left(\varepsilon_{X^{\prime}}\right) \circ \eta_{C\left(X^{\prime}\right)} \\
& =\mathscr{C}\left(\sigma^{\prime} \circ \varphi\right) \circ \mathscr{C} \mathscr{V}\left(\varepsilon_{X^{\prime}}\right) \circ \eta_{C\left(X^{\prime}\right)} \\
& =\mathscr{C}(\mathscr{V}(\varphi) \circ \sigma) \circ \mathscr{C} \mathscr{V}\left(\varepsilon_{X^{\prime}}\right) \circ \eta_{C\left(X^{\prime}\right)} \\
& =\mathscr{C}\left(\mathscr{V}\left(\varepsilon_{X^{\prime}}\right) \circ \mathscr{V}(\varphi) \circ \sigma\right) \circ \eta_{C\left(X^{\prime}\right)} \\
& =\mathscr{C}\left(\mathscr{V}\left(\varepsilon_{X^{\prime}} \circ \varphi\right) \circ \sigma\right) \circ \eta_{C\left(X^{\prime}\right)}
\end{aligned}
$$

On the other hand,

$$
\begin{aligned}
\mathscr{G}_{\sigma} \circ \mathscr{H} \mathscr{C}(\varphi) & =\mathscr{C}(\sigma) \circ \mathscr{C} \mathscr{V}\left(\varepsilon_{X}\right) \circ \eta_{C(X)} \circ \mathscr{H} \mathscr{C}(\varphi) \\
& =\mathscr{C}(\sigma) \circ \mathscr{C} \mathscr{V}\left(\varepsilon_{X}\right) \circ \mathscr{C} \mathscr{V} \mathscr{Y} \mathscr{C}(\varphi) \circ \eta_{C\left(X^{\prime}\right)} \\
& =\mathscr{C}(\sigma) \circ \mathscr{C} \mathscr{V}\left(\mathscr{Y} \mathscr{C}(\varphi) \circ \varepsilon_{X}\right) \circ \eta_{C\left(X^{\prime}\right)} \\
& =\mathscr{C}(\sigma) \circ \mathscr{C} \mathscr{V}\left(\varepsilon_{X^{\prime}} \circ \varphi\right) \circ \eta_{C\left(X^{\prime}\right)} \\
& =\mathscr{C}\left(\mathscr{V}\left(\varepsilon_{X^{\prime}} \circ \varphi\right) \circ \sigma\right) \circ \eta_{C\left(X^{\prime}\right)}
\end{aligned}
$$

where the second equality holds by applying Lemma 6.2 to $\gamma=\mathscr{C}(\varphi)$ and the fourth equality by the naturality of $\varepsilon$. Thus, $\mathscr{C}(\varphi) \circ \mathscr{G}_{\sigma^{\prime}}=\mathscr{G}_{\sigma} \circ \mathscr{H} \mathscr{C}(\varphi)$. It is then straightforward to see that $\mathscr{G}$ is a contravariant functor.

Proposition 6.5. There is a natural isomorphism $\xi: 1_{\mathrm{Coalg}(\mathscr{V})} \rightarrow \mathscr{F} \mathscr{G}$.

Proof. We define $\xi: 1_{\text {Coalg }(\mathscr{V})} \rightarrow \mathscr{F} \mathscr{G}$ as follows. If $(X, \sigma) \in \operatorname{Coalg}(\mathscr{V})$, then $\xi_{(X, \sigma)}=\varepsilon_{X}$.

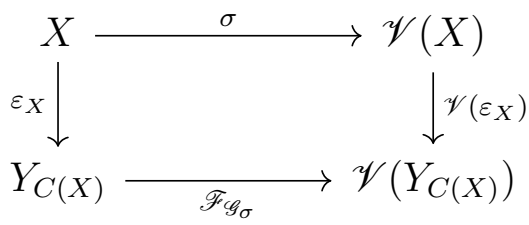

To see that $\varepsilon_{X}$ is a $\operatorname{Coalg}(\mathscr{V})$-morphism, we have $\mathscr{G}_{\sigma}=\mathscr{C}(\sigma) \circ \mathscr{C} \mathscr{V}\left(\varepsilon_{X}\right) \circ \eta_{C(X)}$. Therefore,

$$
\begin{aligned}
\mathscr{F}_{\mathscr{G}_{\sigma}} & =\varepsilon_{\mathscr{V}\left(Y_{C(X)}\right)}^{-1} \circ \mathscr{Y}\left(\eta_{C(X)}\right)^{-1} \circ \mathscr{Y}\left(\mathscr{G}_{\sigma}\right) \\
& =\varepsilon_{\mathscr{V}\left(Y_{C(X)}\right)}^{-1} \circ \mathscr{Y}\left(\eta_{C(X)}\right)^{-1} \circ \mathscr{Y}\left(\mathscr{C}(\sigma) \circ \mathscr{C} \mathscr{V}\left(\varepsilon_{X}\right) \circ \eta_{C(X)}\right) \\
& =\varepsilon_{\mathscr{V}\left(Y_{C(X)}\right)}^{-1} \circ \mathscr{Y}\left(\eta_{C(X)}\right)^{-1} \circ \mathscr{Y}\left(\eta_{C(X)}\right) \circ \mathscr{Y} \mathscr{C} \mathscr{V}\left(\varepsilon_{X}\right) \circ \mathscr{Y} \mathscr{C}(\sigma) \\
& =\varepsilon_{\mathscr{V}\left(Y_{C(X)}\right)}^{-1} \circ \mathscr{Y} \mathscr{C} \mathscr{V}\left(\varepsilon_{X}\right) \circ \mathscr{Y} \mathscr{C}(\sigma) \\
& =\mathscr{V}\left(\varepsilon_{X}\right) \circ \varepsilon_{\mathscr{V}(X)}^{-1} \circ \mathscr{Y} \mathscr{C}(\sigma) \\
& =\mathscr{V}\left(\varepsilon_{X}\right) \circ \sigma \circ \varepsilon_{X}^{-1}
\end{aligned}
$$

where the last two equalities hold since $\varepsilon$ is a natural isomorphism. Composing both sides on the right by $\varepsilon_{X}$ shows that Diagram (4) commutes. Thus, $\varepsilon_{X}$ is a Coalg( $\left.\mathscr{V}\right)$-morphism. 
To see that $\xi: 1_{\operatorname{Coalg}(\mathscr{V})} \rightarrow \mathscr{F} \mathscr{G}$ is a natural transformation, let $\varphi:(X, \sigma) \rightarrow\left(X^{\prime}, \sigma^{\prime}\right)$ be a Coalg $(\mathscr{V})$-morphism. The following diagram commutes since $\varepsilon$ is a natural transformation.

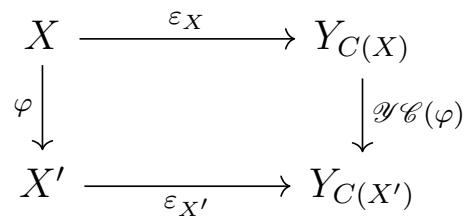

Because $\xi_{(X, \sigma)}=\varepsilon_{X}$ and $\xi_{\left(X^{\prime}, \sigma^{\prime}\right)}=\varepsilon_{X^{\prime}}$, it follows that $\xi$ is natural. It is a natural isomorphism since $\xi_{(X, \sigma)}=\varepsilon_{X}$ is a homeomorphism for each $(X, \sigma) \in \operatorname{Coalg}(\mathscr{V})$.

Proposition 6.6. There is a natural transformation $\kappa: 1_{\mathrm{Alg}(\mathscr{H})} \rightarrow \mathscr{G} \mathscr{F}$.

Proof. We define $\kappa: 1_{\mathrm{Alg}(\mathscr{H})} \rightarrow \mathscr{G} \mathscr{F}$ as follows. Let $(A, \alpha) \in \operatorname{Alg}(\mathscr{H})$. We set $\kappa_{(A, \alpha)}=\zeta_{A}$.

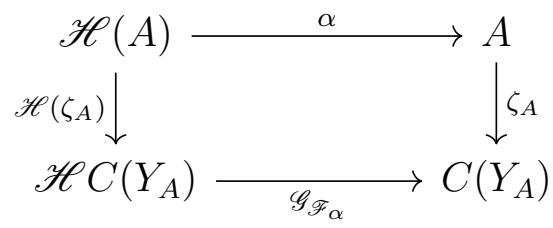

To see that $\zeta_{A}$ is an $\operatorname{Alg}(\mathscr{H})$-morphism, we show that Diagram (5) is commutative. We have $\mathscr{F}_{\alpha}=\varepsilon_{\mathscr{V}\left(Y_{A}\right)}^{-1} \circ \mathscr{Y}\left(\eta_{A}\right)^{-1} \circ \mathscr{Y}(\alpha)$ and so

$$
\begin{aligned}
\mathscr{G}_{\mathscr{F}_{\alpha}} & =\mathscr{C}\left(\mathscr{F}_{\alpha}\right) \circ \mathscr{C} \mathscr{V}\left(\varepsilon_{Y_{A}}\right) \circ \eta_{C\left(Y_{A}\right)} \\
& =\mathscr{C}\left(\varepsilon_{\mathscr{V}\left(Y_{A}\right)}^{-1} \circ \mathscr{Y}\left(\eta_{A}\right)^{-1} \circ \mathscr{Y}(\alpha)\right) \circ \mathscr{C} \mathscr{V}\left(\varepsilon_{Y_{A}}\right) \circ \eta_{C\left(Y_{A}\right)} \\
& =\mathscr{C} \mathscr{Y}(\alpha) \circ \mathscr{C} \mathscr{Y}\left(\eta_{A}\right)^{-1} \circ \mathscr{C}\left(\varepsilon_{\mathscr{V}\left(Y_{A}\right)}\right)^{-1} \circ \mathscr{C} \mathscr{V}\left(\varepsilon_{Y_{A}}\right) \circ \eta_{C\left(Y_{A}\right)} \\
& =\mathscr{C} \mathscr{Y}(\alpha) \circ \mathscr{C} \mathscr{Y}\left(\eta_{A}\right)^{-1} \circ \zeta_{C \mathscr{V}\left(Y_{A}\right)} \circ \mathscr{C} \mathscr{V}\left(\varepsilon_{Y_{A}}\right) \circ \eta_{C\left(Y_{A}\right)} \\
& =\mathscr{C} \mathscr{Y}(\alpha) \circ \mathscr{C} \mathscr{Y}\left(\eta_{A}\right)^{-1} \circ \zeta_{C \mathscr{V}\left(Y_{A}\right)} \circ \mathscr{C} \mathscr{V} \mathscr{Y}\left(\zeta_{A}\right)^{-1} \circ \eta_{C\left(Y_{A}\right)}
\end{aligned}
$$

because $\mathscr{C}\left(\varepsilon_{\mathscr{V}\left(Y_{A}\right)}\right)^{-1}=\zeta_{C \mathscr{V}\left(Y_{A}\right)}$ and $\varepsilon_{Y_{A}}=\mathscr{Y}\left(\zeta_{A}\right)^{-1}$ by Remark 2.5. Thus, by Lemma 6.2 and the naturality of $\zeta$ (used twice),

$$
\begin{aligned}
\mathscr{G}_{\mathscr{F}_{\alpha}} \circ \mathscr{H}\left(\zeta_{A}\right) & =\mathscr{C} \mathscr{Y}(\alpha) \circ \mathscr{C} \mathscr{Y}\left(\eta_{A}\right)^{-1} \circ \zeta_{C \mathscr{V}\left(Y_{A}\right)} \circ \mathscr{C} \mathscr{V} \mathscr{Y}\left(\zeta_{A}\right)^{-1} \circ \eta_{C\left(Y_{A}\right)} \circ \mathscr{H}\left(\zeta_{A}\right) \\
& =\mathscr{C} \mathscr{Y}(\alpha) \circ \mathscr{C} \mathscr{Y}\left(\eta_{A}\right)^{-1} \circ \zeta_{C \mathscr{V}\left(Y_{A}\right)} \circ \mathscr{C} \mathscr{V} \mathscr{Y}\left(\zeta_{A}\right)^{-1} \circ \mathscr{C} \mathscr{V} \mathscr{Y}\left(\zeta_{A}\right) \circ \eta_{A} \\
& =\mathscr{C} \mathscr{Y}(\alpha) \circ \mathscr{C} \mathscr{Y}\left(\eta_{A}\right)^{-1} \circ \zeta_{C \mathscr{V}\left(Y_{A}\right)} \circ \eta_{A} \\
& =\mathscr{C} \mathscr{Y}(\alpha) \circ \zeta_{\mathscr{H}(A)} \\
& =\zeta_{A} \circ \alpha .
\end{aligned}
$$

Thus, $\zeta_{A} \circ \alpha=\mathscr{G}_{\mathscr{F}_{\alpha}} \circ \mathscr{H}\left(\zeta_{A}\right)$, and hence $\zeta_{A}$ is a Coalg $(\mathscr{V})$-morphism.

To show naturality, let $\gamma:(A, \alpha) \rightarrow\left(A^{\prime}, \alpha^{\prime}\right)$ be an $\operatorname{Alg}(\mathscr{H})$-morphism. The following diagram commutes since $\zeta$ is a natural transformation.

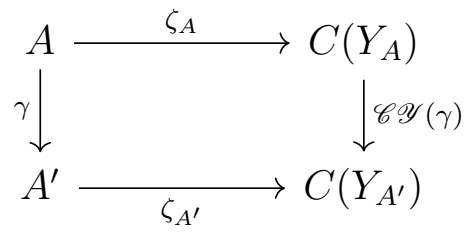


Because $\kappa_{(A, \alpha)}=\zeta_{A}$ and $\kappa_{\left(A^{\prime}, \alpha^{\prime}\right)}=\zeta_{A^{\prime}}$, it follows that $\kappa$ is a natural transformation.

Theorem 6.7. The functors $\mathscr{F}$ and $\mathscr{G}$ yield a dual adjunction between $\mathrm{Alg}(\mathscr{H})$ and $\operatorname{Coalg}(\mathscr{V})$.

Proof. By [20, Thm. IV.1.2] and Propositions 6.3 6.6, it suffices to show that

$$
\mathscr{F}\left(\kappa_{(A, \alpha)}\right) \circ \xi_{\mathscr{F}(A, \alpha)}=1_{\mathscr{F}(A, \alpha)}
$$

and

$$
\mathscr{G}\left(\xi_{(X, \sigma)}\right) \circ \kappa_{\mathscr{G}(X, \sigma)}=1_{\mathscr{G}(X, \sigma)}
$$

for each $(A, \alpha) \in \operatorname{Alg}(\mathscr{H})$ and $(X, \sigma) \in \operatorname{Coalg}(\mathscr{V})$. We have $\kappa_{(A, \alpha)}=\zeta_{A}$ and $\xi_{\mathscr{F}(A, \alpha)}=$ $\varepsilon_{Y_{A}}$. Since $\mathscr{F}\left(\kappa_{(A, \alpha)}\right)=\mathscr{F}\left(\zeta_{A}\right)=\mathscr{Y}\left(\zeta_{A}\right)$ and $1_{\mathscr{F}(A, \alpha)}=1_{Y_{A}}$, the first equation reduces to $\mathscr{Y}\left(\zeta_{A}\right) \circ \varepsilon_{Y_{A}}=1_{Y_{A}}$, which holds by Remark 2.5. For the second equation, $\xi_{(X, \sigma)}=\varepsilon_{X}$ and $\kappa_{\mathscr{G}(X, \sigma)}=\zeta_{C(X)}$. Since $\mathscr{G}\left(\xi_{(X, \sigma)}\right)=\mathscr{G}\left(\varepsilon_{X}\right)=\mathscr{C}\left(\varepsilon_{X}\right)$ and $1_{\mathscr{G}(X, \sigma)}=1_{C(X)}$, the equation $\mathscr{G}\left(\xi_{(X, \sigma)}\right) \circ \kappa_{\mathscr{G}(X, \sigma)}=1_{\mathscr{G}(X, \sigma)}$ is equivalent to $\mathscr{C}\left(\varepsilon_{X}\right) \circ \zeta_{C(X)}=1_{C(X)}$, which also holds by Remark 2.5. Therefore, $\mathscr{F}$ and $\mathscr{G}$ form a dual adjunction.

Definition 6.8. $\operatorname{Let} \operatorname{Alg}^{u}(\mathscr{H})$ be the full subcategory of $\operatorname{Alg}(\mathscr{H})$ consisting of those $(A, \alpha)$ with $A \in \boldsymbol{u b a \ell}$.

\section{Corollary 6.9.}

(1) The functors $\mathscr{F}$ and $\mathscr{G}$ restrict to a dual equivalence between $\operatorname{Alg}^{u}(\mathscr{H})$ and $\operatorname{Coalg}(\mathscr{V})$.

(2) $\operatorname{Alg}^{u}(\mathscr{H})$ is a reflective subcategory of $\operatorname{Alg}(\mathscr{H})$.

Proof. (1) Let $(A, \alpha) \in \operatorname{Alg}(\mathscr{H})$. Then $\kappa_{(A, \alpha)}=\zeta_{A}$ is an isomorphism iff $A \in \boldsymbol{u b a} \boldsymbol{\ell}$ iff $(A, \alpha) \in$ $\operatorname{Alg}^{u}(\mathscr{H})$. Consequently, $\kappa: 1_{\mathrm{Alg}^{u}(\mathscr{H})} \rightarrow \mathscr{G} \mathscr{F}$ is a natural isomorphism by Proposition 6.6. Moreover, $\xi$ is a natural isomorphism by Proposition 6.5. Therefore, $\mathscr{F}$ and $\mathscr{G}$ restrict to a dual equivalence between $\operatorname{Alg}^{u}(\mathscr{H})$ and $\operatorname{Coalg}(\mathscr{V})$ by [20, Thm. IV.4.1].

$(2)$ By (1), the functors $\mathscr{F}$ and $\mathscr{G}$ form a dual equivalence between $\operatorname{Alg}^{u}(\mathscr{H})$ and $\operatorname{Coalg}(\mathscr{V})$. If $(A, \alpha) \in \operatorname{Alg}(\mathscr{H})$, then the morphism $\kappa_{(A, \alpha)}$ is a universal arrow from $(A, \alpha)$ to $\mathscr{F}$ by 20 , Thm. IV.1.1]. Therefore, $\operatorname{Alg}^{u}(\mathscr{H})$ is a reflective subcatgory of $\operatorname{Alg}(\mathscr{H})$ (see [20, p. 89]).

Proposition 6.10. The functors $\mathscr{M}, \mathscr{N}$ yield an isomorphism between $\operatorname{Alg}^{u}(\mathscr{H})$ and $\mathbf{m u b a} \boldsymbol{\ell}$.

Proof. If $(A, \sigma) \in \operatorname{Alg}^{u}(\mathscr{H})$, then $A \in \boldsymbol{u b a} \boldsymbol{\ell}$, so $\mathscr{M}(A, \sigma)=\left(A, \square_{\sigma}\right) \in \boldsymbol{m u b a} \boldsymbol{\ell}$. If $(A, \square) \in$ muba $\boldsymbol{\ell}$, then $A \in \boldsymbol{u b a} \boldsymbol{\ell}$, so $\mathscr{N}(A, \square)=\left(A, \sigma_{\square}\right) \in \operatorname{Alg}^{u}(\mathscr{H})$. Therefore, the proof of Theorem 4.5 shows that $\mathscr{M}$ and $\mathscr{N}$ restrict to $\operatorname{Alg}^{u}(\mathscr{H})$ and $\boldsymbol{m u b a} \boldsymbol{\ell}$, respectively, to yield an isomorphism.

We finish this section by giving an alternate view of the category $\operatorname{Alg}^{u}(\mathscr{H})$.

Definition 6.11. We let $\mathscr{H}^{u}$ be the endofunctor $\mathscr{C} \mathscr{Y} \mathscr{H}$ on $\boldsymbol{u b a} \boldsymbol{\ell}$. Therefore, if $A \in \boldsymbol{u b a} \boldsymbol{\ell}$, then $\mathscr{H}^{u}(A)=C\left(Y_{\mathscr{H}(A)}\right)$ and if $\alpha: A \rightarrow A^{\prime}$ is a $\boldsymbol{u} \boldsymbol{b a} \boldsymbol{\ell}$-morphism, then $\mathscr{H}^{u}(\alpha)=\mathscr{C} \mathscr{Y} \mathscr{H}(\alpha)$. 
Recall from Section 2.1 that if $\gamma: A \rightarrow B$ is a $\boldsymbol{b a} \boldsymbol{\ell}$-morphism with $B \in \boldsymbol{u} \boldsymbol{b a} \boldsymbol{\ell}$, then there is a unique $\boldsymbol{b a} \boldsymbol{\ell}$-morphism $\gamma^{u}: C\left(Y_{A}\right) \rightarrow B$ with $\gamma^{u} \circ \zeta_{A}=\gamma$, where $\gamma^{u}=\zeta_{B}^{-1} \circ \mathscr{C} \mathscr{Y}(\gamma)$.

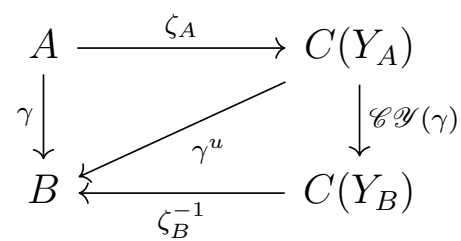

Proposition 6.12. There is an isomorphism of categories between $\operatorname{Alg}^{u}(\mathscr{H})$ and $\operatorname{Alg}\left(\mathscr{H}^{u}\right)$.

Proof. We define $\mathscr{A}: \operatorname{Alg}^{u}(\mathscr{H}) \rightarrow \operatorname{Alg}\left(\mathscr{H}^{u}\right)$ on objects by sending $(A, \alpha)$ to $\left(A, \alpha^{u}\right)$. On morphisms, if $\gamma$ is an $\operatorname{Alg}(\mathscr{H})$-morphism, then $\mathscr{A}(\gamma)=\gamma$.

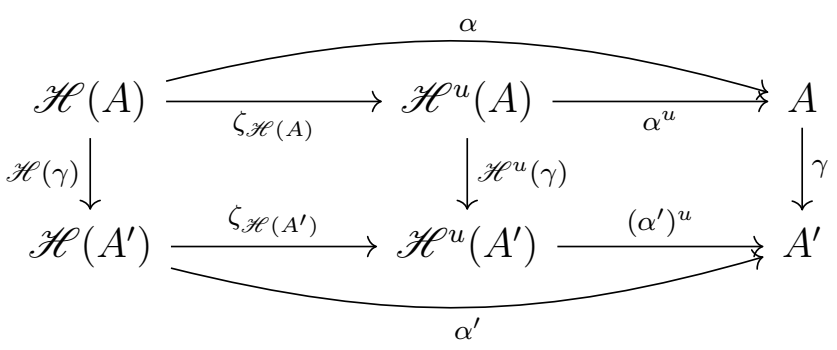

To see that $\gamma$ is an $\operatorname{Alg}\left(\mathscr{H}^{u}\right)$-morphism, the left square of the diagram commutes by the naturality of $\zeta$. We have

$$
\left(\gamma \circ \alpha^{u}\right) \circ \zeta_{\mathscr{H}(A)}=\gamma \circ \alpha=\alpha^{\prime} \circ \mathscr{H}(\gamma)=\left(\alpha^{\prime}\right)^{u} \circ \zeta_{\mathscr{H}\left(A^{\prime}\right)} \circ \mathscr{H}(\gamma)=\left(\alpha^{\prime}\right)^{u} \circ \mathscr{H}^{u}(\gamma) \circ \zeta_{\mathscr{H}}(A)
$$

so $\gamma \circ \alpha^{u}=\left(\alpha^{\prime}\right)^{u} \circ \mathscr{H}^{u}(\gamma)$ since $\zeta_{\mathscr{H}(A)}$ is epic. This shows that $\gamma$ is an $\operatorname{Alg}\left(\mathscr{H}^{u}\right)$-morphism. It then follows that $\mathscr{A}$ is a covariant functor.

Going in the opposite direction, we define a functor $\mathscr{B}: \operatorname{Alg}\left(\mathscr{H}^{u}\right) \rightarrow \operatorname{Alg}^{u}(\mathscr{H})$ on objects by sending $(A, \alpha)$ to $\left(A, \alpha \circ \zeta_{\mathscr{H}(A)}\right)$. On morphisms we send a $\operatorname{Alg}\left(\mathscr{H}^{u}\right)$-morphism $\gamma: A \rightarrow A^{\prime}$ to itself. It is clear that $\mathscr{B}$ is a covariant functor.

If $(A, \alpha) \in \operatorname{Alg}^{u}(\mathscr{H})$, then $\mathscr{A}(A, \alpha)=\left(A, \alpha^{u}\right)$, and so $\mathscr{B} \mathscr{A}(A, \alpha)=\left(A, \alpha^{u} \circ \zeta_{\mathscr{H}}(A)\right)=$ $(A, \alpha)$. Therefore, $\mathscr{B} \mathscr{A}=1_{\operatorname{Alg}^{u}(\mathscr{H})}$. If $(A, \alpha) \in \operatorname{Alg}\left(\mathscr{H}^{u}\right)$, then $(A, \alpha \circ \zeta \mathscr{H}(A)) \in \operatorname{Alg}^{u}(\mathscr{H})$, and $\left(\alpha \circ \zeta_{\mathscr{H}(A)}\right)^{u}=\alpha$. Therefore, $\mathscr{A} \mathscr{B}=1_{\mathrm{Alg}(\mathscr{H} u)}$. Thus, $\mathscr{A}, \mathscr{B}$ yield an isomorphism of categories between $\operatorname{Alg}^{u}(\mathscr{H})$ and $\operatorname{Alg}\left(\mathscr{H}^{u}\right)$.

\section{7. $\boldsymbol{m b a} \ell$ AND KHF}

In this section we show how to derive from our results the dual adjunction between $\boldsymbol{m b a} \boldsymbol{\ell}$ and KHF and the dual equivalence between muba $\boldsymbol{\ell}$ and KHF obtained in [7].

We start by recalling (see, e.g., [5, Thm. 2.16]) that there is an isomorphism of categories between Coalg $(\mathscr{V})$ and KHF. The isomorphism is determined by the following functors. The functor $\mathscr{S}: \operatorname{Coalg}(\mathscr{V}) \rightarrow \operatorname{KHF}$ sends $(X, \sigma)$ to $\left(X, R_{\sigma}\right) \in \mathrm{KHF}$, where $x R_{\sigma} y$ if $y \in \sigma(x)$, and $\mathscr{S}$ sends a $\operatorname{Coalg}(\mathscr{V})$ morphism to itself. The functor $\mathscr{T}: \operatorname{KHF} \rightarrow \operatorname{Coalg}(\mathscr{V})$ sends $(X, R) \in \mathrm{KHF}$ to $\left(X, \sigma_{R}\right)$, defined by $\sigma_{R}(x)=R[x]$, and sends a KHF-morphism to itself.

As a consequence of this and the results of the previous section, we obtain the main result of [7]. 
Theorem 7.1. [7, Thm. 5.3] There is a dual adjunction between mbal and KHF which restricts to a dual equivalence between mubal and KHF.

Proof. By Theorem 6.7 the functors $\mathscr{F}$ and $\mathscr{G}$ form a dual adjunction between $\mathrm{Alg}(\mathscr{H})$ and Coalg $(\mathscr{V})$. By Theorem 4.5, the functors $\mathscr{M}, \mathscr{N}$ yield an isomorphism of categories between $\operatorname{Alg}(\mathscr{H})$ and $\boldsymbol{m b a} \boldsymbol{\ell}$. The functors $\mathscr{S}, \mathscr{T}$ yield an isomorphism of categories between Coalg $(\mathscr{V})$ and KHF [5, Thm. 2.16]. We thus have the following diagram.

$$
\text { mubal } \longleftrightarrow \text { mbal } \underset{\mathscr{M}}{\stackrel{\mathscr{N}}{\rightleftarrows}} \operatorname{Alg}(\mathscr{H}) \underset{\mathscr{F}}{\stackrel{\mathscr{F}}{\rightleftarrows}} \operatorname{Coalg}(\mathscr{V}) \underset{\mathscr{T}}{\stackrel{\mathscr{S}}{\rightleftarrows}} \mathrm{KHF}
$$

Consequently, $\mathscr{S} \mathscr{F} \mathscr{N}: \boldsymbol{m b a} \ell \rightarrow \mathrm{KHF}$ and $\mathscr{M} \mathscr{G} \mathscr{T}: \mathrm{KHF} \rightarrow \boldsymbol{m b a} \ell$ yield a dual adjunction which restricts to a dual equivalence between $\boldsymbol{m u b a \ell}$ and KHF.

Proposition 7.2. $\mathscr{S} \mathscr{F} \mathscr{N}$ and $\mathscr{M} \mathscr{G} \mathscr{T}$ are precisely the functors $\mathscr{C}$ and $\mathscr{Y}$ yielding the dual adjunction of [7, Thm. 5.2].

Proof. Let $(A, \square) \in \boldsymbol{m b a} \boldsymbol{l}$. Then $\mathscr{Y}(A, \square)=\left(Y_{A}, R_{\square}\right)$, where we recall from Section 2.3 that $R_{\square}$ is defined by $x R_{\square} y$ if $y^{+} \subseteq \square^{-1} x$. We have $\mathscr{N}(A, \square)=\left(A, \sigma_{\square}\right)$, which satisfies $\sigma_{\square}\left(\square_{a}\right)=\square a$ for all $a \in A$. Then $\mathscr{F}\left(A, \sigma_{\square}\right)=\left(Y_{A}, \mathscr{F}_{\sigma_{\square}}\right)$, where we recall that $\mathscr{F}_{\sigma_{\square}}=$ $\varepsilon_{\mathscr{V}\left(Y_{A}\right)}^{-1} \circ \mathscr{Y}\left(\eta_{A}\right)^{-1} \circ \mathscr{Y}\left(\sigma_{\square}\right)$. Finally, $\mathscr{S}$ sends this to $\left(Y_{A}, R_{\mathscr{F}_{\sigma_{\square}}}\right)$, where $x R_{\mathscr{F}_{\sigma_{\square}}} y$ if $y \in$ $\mathscr{F}_{\sigma_{\square}}(x)$. Let $x \in Y_{A}$ and $F=\mathscr{F}_{\sigma_{\square}}(x) \in \mathscr{V}\left(Y_{A}\right)$. If $M=\varepsilon_{\mathscr{V}\left(Y_{A}\right)}(F) \in Y_{C\left(\mathscr{V} Y_{A}\right)}$, then $M=\left\{g \in C\left(\mathscr{V} Y_{A}\right) \mid g(F)=0\right\}$ and

$$
\mathscr{Y}\left(\eta_{A}\right)(M)=\eta_{A}^{-1}(M)=\sigma_{\square}^{-1}(x)=\mathscr{Y}\left(\sigma_{\square}\right)(x) .
$$

We show that $R_{\square}=R_{\mathscr{F}_{\sigma_{\square}}}$. Suppose that $x R_{\square} y$, so $\square y^{+} \subseteq x$. To see that $x R_{\mathscr{F}_{\sigma}} y$, we need to show that $y \in F$. If not, then by Urysohn's lemma and the fact that $\zeta_{A}[A]$ is uniformly dense in $C\left(Y_{A}\right)$, there is $a \in A$ with $\zeta_{A}(a)(y)=0$ and $\zeta_{A}(a)[F] \geq 1 / 2$. By replacing $a$ by $a^{+}$we may assume that $a \geq 0$. Since $\zeta_{A}(a)(y)=0$, we have $a \in y$. Therefore, $\square a \in x$. This means $\sigma_{\square}\left(\square_{a}\right) \in x$, so $\square_{a} \in \sigma_{\square}^{-1}(x)=\eta_{A}^{-1}(M)$. Thus, $\eta_{A}\left(\square_{a}\right) \in M$, so $g_{A}(a) \in M$. Therefore, $\inf g_{A}\left(\zeta_{A}(a)\right)(F)=0$, which is false by construction of $a$. This shows $y \in F$.

Conversely, if $x R_{\mathscr{F}_{\sigma}} y$, then $y \in F$. Let $a \in y^{+}$. Then inf $g_{A}\left(\zeta_{A}(a)\right)(F)=0$ because $a \in y$ and $a \geq 0$. Therefore, $\eta_{A}\left(\square_{a}\right) \in M$, so $\square_{a} \in \eta_{A}^{-1}(M)=\sigma_{\square}^{-1}(x)$. Thus, $\square a=\sigma_{\square}\left(\square_{a}\right) \in x$. This shows $\square y^{+} \subseteq x$, so $x R_{\square} y$. This completes the proof that $R_{\mathscr{F}_{\sigma_{\square}}}=R_{\square}$. Therefore, $\mathscr{Y}$ and $\mathscr{S} \mathscr{F} \mathscr{N}$ agree on the objects of $\boldsymbol{m b a} \boldsymbol{\ell}$. For morphisms, if $\alpha:(A, \square) \rightarrow\left(A^{\prime}, \square^{\prime}\right)$ is an mba $\boldsymbol{\ell}$-morphism, then $\mathscr{S} \mathscr{F} \mathscr{N}(\alpha)=\mathscr{S} \mathscr{F}(\alpha)=\mathscr{S}(\mathscr{Y}(\alpha))=\mathscr{Y}(\alpha)$. Thus, $\mathscr{S} \mathscr{F} \mathscr{N}=\mathscr{Y}$.

In the opposite direction, if $(X, R) \in \mathrm{KHF}$, we show that $\mathscr{C}(X, R)=\mathscr{M} \mathscr{G} \mathscr{T}(X, R)$. First, $\mathscr{C}(X, R)=\left(C(X), \square_{R}\right)$, where we recall from Section 2.3 that $\square_{R} f$ is given by

$$
\left(\square_{R} f\right)(x)=\left\{\begin{array}{cl}
\inf f R[x] & \text { if } R[x] \neq \varnothing \\
1 & \text { if } R[x]=\varnothing .
\end{array}\right.
$$

The functor $\mathscr{T}$ sends $(X, R)$ to $\left(X, \sigma_{R}\right)$, where $\sigma_{R}(x)=R[x]$. Then $\mathscr{G}$ sends this to $\left(C(X), \mathscr{G}_{\sigma_{R}}\right)$, where we recall that $\mathscr{G}_{\sigma_{R}}=\mathscr{C}\left(\sigma_{R}\right) \circ \mathscr{C} \mathscr{V}\left(\varepsilon_{X}\right) \circ \eta_{C(X)}$. Finally, $\left(C(X), \mathscr{G}_{\sigma_{R}}\right)$ 
is sent by $\mathscr{M}$ to $\left(C(X), \square_{\mathscr{G}_{\sigma_{R}}}\right)$, where $\square_{\mathscr{G}_{\sigma_{R}}} f=\mathscr{G}_{\sigma_{R}}\left(\square_{f}\right)$. We have

$$
\begin{aligned}
\mathscr{G}_{\sigma_{R}}\left(\square_{f}\right) & =\mathscr{C}\left(\sigma_{R}\right)\left(\mathscr{C} \mathscr{V}\left(\varepsilon_{X}\right)\left(\eta_{C(X)}\left(\square_{f}\right)\right)\right) \\
& =\mathscr{C}\left(\sigma_{R}\right)\left(\mathscr{C} \mathscr{V}\left(\varepsilon_{X}\right)\left(g_{C(X)}(f)\right)\right) \\
& =\mathscr{C}\left(\sigma_{R}\right)\left(g_{C(X)}(f) \circ \mathscr{V}\left(\varepsilon_{X}\right)\right) \\
& =g_{C(X)}(f) \circ \mathscr{V}\left(\varepsilon_{X}\right) \circ \sigma_{R} .
\end{aligned}
$$

Let $x \in X$. Then $\sigma_{R}(x)=R[x]$ and $\mathscr{V}\left(\varepsilon_{X}\right)(R[x])=\varepsilon_{X}(R[x])$. Therefore, since $f=$ $\zeta_{C(X)}(f) \circ \varepsilon_{X}$ by Remark 2.5, we have

$$
\begin{aligned}
g_{C(X)}(f)\left(\varepsilon_{X} R[x]\right) & =\left\{\begin{array}{cl}
\inf \zeta_{C(X)}(f)\left(\varepsilon_{X} R[x]\right) & \text { if } R[x] \neq \varnothing \\
1 & \text { if } R[x]=\varnothing
\end{array}\right. \\
& =\left\{\begin{array}{cl}
\inf f R[x] & \text { if } R[x] \neq \varnothing \\
1 & \text { if } R[x]=\varnothing
\end{array}\right. \\
& =\left(\square_{R} f\right)(x) .
\end{aligned}
$$

Thus, $\mathscr{C}$ and $\mathscr{M} \mathscr{G} \mathscr{T}$ agree on objects of KHF. If $\sigma:(X, R) \rightarrow\left(X^{\prime}, R^{\prime}\right)$ is a KHF-morphism, then $\mathscr{M} \mathscr{G} \mathscr{T}(\sigma)=\mathscr{M} \mathscr{C}(\sigma)=\mathscr{C}(\sigma)$. Consequently, $\mathscr{M} \mathscr{G} \mathscr{T}=\mathscr{C}$.

We conclude this section with the following diagram showing the relationship between the various categories we have considered, where the curved vertical arrows are reflections.

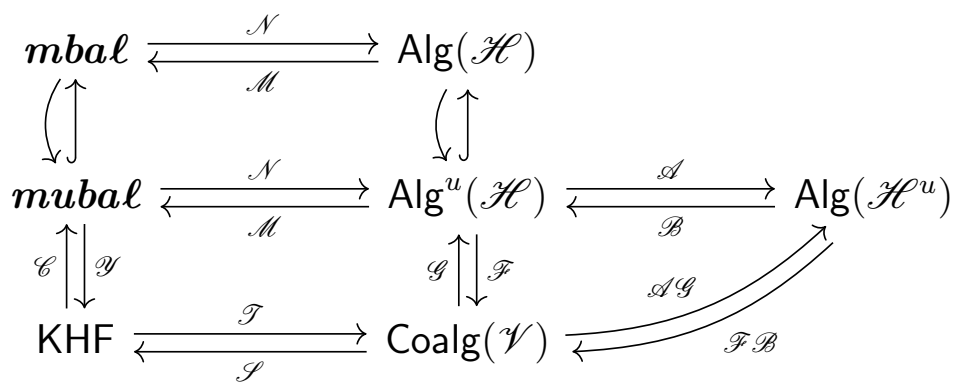

\section{THE SERIAL CASE}

We recall that a binary relation $R$ on a set $X$ is serial provided that $R[x] \neq \varnothing$ for each $x \in X$. If $(X, R) \in \mathrm{KHF}$ with $R$ serial, then we can replace $\mathscr{V}(X)$ with $\mathscr{V}^{*}(X)$ in our considerations. It was shown in [7, Prop. 7.2] that the binary relation $R_{\square}$ in the dual compact Hausdorff frame of $(A, \square) \in \boldsymbol{m b a} \boldsymbol{\ell}$ is serial iff $\square 0=0$. We thus arrive at the following definition, the notation of which is motivated by modal logic, where the seriality axiom is denoted by $\mathrm{D}$ (see, e.g., [11]).

Definition 8.1. [7, Sec. 7]

(1) Let $\boldsymbol{m} \boldsymbol{b} \boldsymbol{a} \boldsymbol{\ell}^{\mathrm{D}}$ be the full subcategory of $\boldsymbol{m} \boldsymbol{b a} \boldsymbol{\ell}$ consisting of those $(A, \square) \in \boldsymbol{m} \boldsymbol{b a} \boldsymbol{\ell}$ with $\square 0=0$.

(2) Let $\boldsymbol{m} \boldsymbol{u} \boldsymbol{b} \boldsymbol{a} \boldsymbol{\ell}^{\mathrm{D}}$ be the full subcategory of $\boldsymbol{m} \boldsymbol{b} \boldsymbol{a} \boldsymbol{\ell}^{\mathrm{D}}$ consisting of those $(A, \square) \in \boldsymbol{m} \boldsymbol{b} \boldsymbol{a} \boldsymbol{\ell}^{\mathrm{D}}$ with $A \in \boldsymbol{u b a \ell}$. 
(3) Let $\mathrm{KHF}^{\mathrm{D}}$ be the full subcategory of $\mathrm{KHF}$ consisting of those $(X, R)$ for which $R$ is a serial relation.

As was pointed out in [7, Rem. 3.12], when $\square 0=0$, the axioms (M2), (M4), and (M5) simplify to the following axioms:

$\left(\mathrm{M}^{*}\right) \square r=r$.

$\left(\mathrm{M}^{*}\right) \square(a+r)=\square a+r$.

$\left(\mathrm{M}^{*}\right) \square(r a)=r(\square a)$ provided $r \geq 0$.

Moreover, (M2*) follows from (M4*) by setting $a=0$.

In [7, Sec. 7] we showed that the functors $\mathscr{C}$ and $\mathscr{Y}$ restrict to yield a dual adjunction between $\boldsymbol{m} \boldsymbol{b a} \ell^{\mathrm{D}}$ and $\mathrm{KHF}^{\mathrm{D}}$, which further restricts to a dual equivalence between $\boldsymbol{m} \boldsymbol{u} \boldsymbol{b a} \boldsymbol{\ell}^{\mathrm{D}}$ and $\mathrm{KHF}^{\mathrm{D}}$. In this section we briefly outline how to derive this result from our considerations by simplifying the definition of $\mathscr{H}$ to produce a functor $\mathscr{H}^{*}$ such that $\operatorname{Alg}\left(\mathscr{H}^{*}\right)$ is isomorphic to $m b a \ell^{\mathrm{D}}$.

Definition 8.2. Let $A \in \boldsymbol{b a \ell}$.

(1) Let $I_{A}^{*}$ be the archimedean $\ell$-ideal of $F(A)$ generated by the following classes of elements.

(a): $f_{A}(a \wedge b)-f_{A}(a) \wedge f_{A}(b)$;

(c): $f_{A}\left(a^{+}\right)-f_{A}(a)^{+}$;

$\left(\mathbf{d}^{*}\right): f_{A}(a+r)-f_{A}(a)-r$;

$\left(\mathbf{e}^{*}\right): f_{A}(r a)-r f_{A}(a)$ if $0 \leq r$.

(2) Let $\mathscr{H}^{*}(A)=F(A) / I_{A}^{*}$ and $h_{A}^{*}: A \rightarrow \mathscr{H}^{*}(A)$ be the composition of $f_{A}^{*}$ with the quotient map $\pi: F(A) \rightarrow \mathscr{H}^{*}(A)$.

(3) For $a \in A$ let $\square_{a}^{*}=h_{A}^{*}(a)$.

(4) Let $\mathscr{V}^{*}$ be the endofunctor on KHaus that sends $X$ to the subspace $\mathscr{V}^{*}(X)=\mathscr{V}(X) \backslash$ $\{\varnothing\}$ of $\mathscr{V}(X)$.

The table below compares the relations in $\mathscr{H}^{*}(A)$ to those of $\mathscr{H}(A)$.

\begin{tabular}{|l|l|}
\hline Relations for $\mathscr{H}^{*}(A)$ & Relations for $\mathscr{H}(A)$ \\
\hline (1) $\square_{a \wedge b}^{*}=\square_{a}^{*} \wedge \square_{b}^{*}$ & (1) $\square_{a \wedge b}=\square_{a} \wedge \square_{b}$ \\
(2) - (2) $\square_{r}=r+(1-r) \square_{0}$ \\
(3) $\square_{a^{+}}^{*}=\left(\square_{a}^{*}\right)^{+}$ & (3) $\square_{a^{+}}=\left(\square_{a}\right)^{+}$ \\
(4) $\square_{a+r}^{*}=\square_{a}^{*}+r$ & (4) $\square_{a+r}=\square_{a}+\square_{r}-\square_{0}$ \\
(5) $\square_{r a}^{*}=r \square_{a}^{*}$ if $0 \leq r$ & (5) $\square_{r a}=\square_{r} \square_{a}$ if $0 \leq r$ \\
\hline
\end{tabular}

\section{Remark 8.3.}

(1) If we set $r=0$ in (5) we see that $\square_{0}^{*}=0$. Furthermore, setting $a=0$ in (4) yields $\square_{r}^{*}=r$. 
(2) From the relations above it follows that $\mathscr{H}^{*}(A)$ is the quotient of $\mathscr{H}(A)$ by the archimedian $\ell$-ideal of $\mathscr{H}(A)$ generated by $\square_{0}$. Consequently, if $(A, \alpha) \in \operatorname{Alg}(\mathscr{H})$ with $\alpha\left(\square_{0}\right)=0$, then there is an induced object $\left(A, \alpha^{*}\right) \in \operatorname{Alg}\left(\mathscr{H}^{*}\right)$.

Theorem 8.4. There is an isomorphism of categories between $\mathrm{Alg}\left(\mathscr{H}^{*}\right)$ and $\boldsymbol{m b a} \boldsymbol{\ell}^{\mathrm{D}}$.

Proof. The functor $\operatorname{Alg}\left(\mathscr{H}^{*}\right) \rightarrow \boldsymbol{m b a} \ell^{\mathrm{D}}$ is defined essentially the same as in Lemma 4.3. To define the functor in the other direction, if $(A, \square) \in \boldsymbol{m} \boldsymbol{b a} \boldsymbol{\ell}^{\mathrm{D}}$, then the induced $\boldsymbol{b} \boldsymbol{a} \boldsymbol{\ell}$ morphism $\sigma_{\square}: \mathscr{H}(A) \rightarrow A$ satisfies $\sigma_{\square}\left(\square_{0}\right)=0$, so induces an object $\left(A, \sigma_{\square}^{*}\right) \in \operatorname{Alg}\left(\mathscr{H}^{*}\right)$ by Remark $\left[8.3(2)\right.$, which satisfies $\sigma_{\square}^{*}\left(\square_{a}^{*}\right)=\square a$ for each $a \in A$. This gives the functor $\boldsymbol{m b a} \ell^{\mathrm{D}} \rightarrow \operatorname{Alg}\left(\mathscr{H}^{*}\right)$. The proof that these functors yield an isomorphism is essentially the same as that of Theorem 4.5.

In parallel with Definition 6.8, $\operatorname{let} \operatorname{Alg}^{u}\left(\mathscr{H}^{*}\right)$ be the full subcategory of $\operatorname{Alg}\left(\mathscr{H}^{*}\right)$ consisting of those $(A, \alpha)$ with $A \in \boldsymbol{u b a \ell}$. The proof of the following result is similar to that of Theorem 6.7 and Corollary $6.9(1)$, with small changes similar to those of the previous theorem. We therefore leave out the details.

Theorem 8.5. There is a dual adjunction between $\operatorname{Alg}\left(\mathscr{H}^{*}\right)$ and $\operatorname{Coalg}\left(\mathscr{V}^{*}\right)$ which restricts to a dual equivalence between $\operatorname{Alg}^{u}\left(\mathscr{H}^{*}\right)$ and $\operatorname{Coalg}\left(\mathscr{V}^{*}\right)$.

Theorem 8.6. There is a dual adjunction between $\boldsymbol{m b a} \boldsymbol{\ell}^{\mathrm{D}}$ and $\mathrm{KHF}^{\mathrm{D}}$ which restricts to a dual equivalence between muba $\ell^{\mathrm{D}}$ and $\mathrm{KHF}^{\mathrm{D}}$.

Proof. The proof is similar to that of Theorem 7.1 but uses Theorem 8.5 instead of Theorem 6.7 and Corollary 6.9)(1). It also uses the isomorphism between $\operatorname{Coalg}\left(\mathscr{V}^{*}\right)$ and $\mathrm{KHF}^{\mathrm{D}}$, which is essentially the same as that between $\operatorname{Coalg}(\mathscr{V})$ and KHF. To give some detail, if $(X, \sigma) \in \operatorname{Coalg}\left(\mathscr{V}^{*}\right)$, then (by abusing notation) $\mathscr{S}$ sends it to $\left(X, R_{\sigma}\right)$. Since the image of $\sigma$ is in $\mathscr{V}^{*}(X)$, the relation $R_{\sigma}$ is serial, so $\left(X, R_{\sigma}\right) \in \mathrm{KHF}^{\mathrm{D}}$. Conversely, if $(X, R) \in \mathrm{KHF}^{\mathrm{D}}$, then $R$ is serial, so $\sigma_{R}(x)=R[x] \neq \varnothing$ for each $x \in X$, so $\sigma_{R}: X \rightarrow \mathscr{V}^{*}(X)$ is continuous, and hence (again abusing notation) $\mathscr{T}(X, R)=\left(X, \sigma_{R}\right) \in \operatorname{Coalg}\left(\mathscr{V}^{*}\right)$.

Remark 8.7. A slight change in the argument of Proposition 6.12 shows that $\operatorname{Alg}^{u}\left(\mathscr{H}^{*}\right)$ is isomorphic to $\operatorname{Alg}\left(\mathscr{H}^{* u}\right)$, where $\mathscr{H}^{* u}$ is the composition $\mathscr{C} \mathscr{Y} \mathscr{H}^{*}$.

\section{Connection to modal Algebras And Descriptive FRAmes}

In this final section we connect our results with those of Abramsky [1] and Kupke, Kurz, and Venema [19]. We start by recalling the definition of those $A \in \boldsymbol{b} \boldsymbol{a} \boldsymbol{\ell}$ that are clean as rings (see, e.g., [21] and the references therein).

Definition 9.1. We call $A \in \boldsymbol{b a} \boldsymbol{\ell}$ clean if each $a \in A$ can be written as $a=e+v$ with $e$

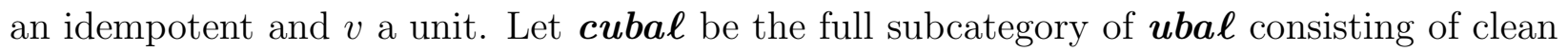
rings.

Lemma 9.2. If $A \in \mathbf{c u b a \ell}$, then $\mathscr{H}^{u}(A) \in \boldsymbol{c u b a \ell}$. 
Proof. By [8, Prop. 5.20], if $A \in \boldsymbol{c u b a \ell}$, then $Y_{A}$ is a Stone space. Therefore, $\mathscr{V}\left(Y_{A}\right)$ is a Stone space, and hence $Y_{\mathscr{H}^{u}(A)}$ is a Stone space by Theorem 5.8. Thus, $\mathscr{H}^{u}(A) \in \boldsymbol{c u b a \ell}$ by [8, Prop. 5.20].

To distinguish between $\mathscr{V}$ on KHaus and Stone, we denote the Vietoris endofunctor on Stone by $\mathscr{V}^{\mathrm{S}}$. By Lemma $9.2, \mathscr{H}^{u}$ restricts to an endofunctor on $\boldsymbol{c u b a} \boldsymbol{\ell}$, which we denote by $\mathscr{H}^{c}$. The following result is then an immediate consequence of Corollary $6.9(1)$.

Theorem 9.3. There is a dual equivalence between $\operatorname{Alg}^{u}\left(\mathscr{H}^{c}\right)$ and $\operatorname{Coalg}\left(\mathscr{V}^{\mathrm{S}}\right)$.

We let $\mathscr{H}^{\mathrm{BA}}$ be the functor of [19] that sends $B \in \mathrm{BA}$ to the free boolean algebra over its underlying meet-semilattice. It was shown in [19, Prop., 3.12] that $\operatorname{Alg}\left(\mathscr{H}^{\mathrm{BA}}\right)$ is isomorphic to the category MA of modal algebras. In parallel of $\mathscr{M}: \operatorname{Alg}(\mathscr{H}) \rightarrow \boldsymbol{m b a} \boldsymbol{\ell}$ and $\mathscr{N}: \boldsymbol{m b a} \boldsymbol{l} \rightarrow$ $\mathrm{Alg}(\mathscr{H})$, we denote the functors giving the isomorphism by $\mathscr{M}^{\mathrm{BA}}: \operatorname{Alg}\left(\mathscr{H}^{\mathrm{BA}}\right) \rightarrow \mathrm{MA}$ and $\mathscr{N}^{\mathrm{BA}}: \mathrm{MA} \rightarrow \operatorname{Alg}\left(\mathscr{H}^{\mathrm{BA}}\right)$. By [7, Thm. 6.9], the triangle in the diagram below commutes up to natural isomorphism, where $(-)^{*}: \mathrm{DF} \rightarrow \mathrm{MA}$ and $(-)_{*}: \mathrm{MA} \rightarrow \mathrm{DF}$ are the functors yielding Esakia-Goldblatt duality, and the functor Id sends $(A, \square) \in \boldsymbol{m b a} \boldsymbol{\ell}$ to $\left(\operatorname{Id}(A),\left.\square\right|_{\operatorname{Id}(A)}\right)$ (see [7, Lem. 6.5]). Therefore, there is an equivalence of categories between $\operatorname{Alg}\left(\mathscr{H}^{c}\right)$ and $\operatorname{Alg}\left(\mathscr{H}^{\mathrm{BA}}\right)$, where the functor $\operatorname{Alg}\left(\mathscr{H}^{c}\right) \rightarrow \operatorname{Alg}\left(\mathscr{H}^{\mathrm{BA}}\right)$ is the composition $\mathscr{N}^{\mathrm{BA}} \circ \operatorname{Id} \circ \mathscr{M}$.

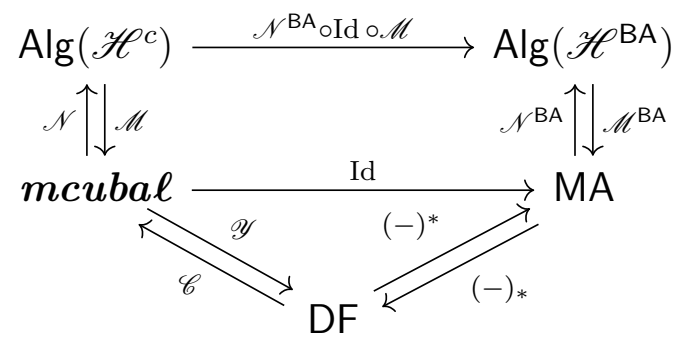

\section{REFERENCES}

[1] S. Abramsky, A Cook's tour of the finitary non-well-founded sets, Invited Lecture at BCTCS, 1988. Available at arXiv:1111.7148.

[2] J. Adámek, H. Herrlich, and G. E. Strecker, Abstract and concrete categories: the joy of cats, Repr. Theory Appl. Categ. (2006), no. 17, 1-507.

[3] B. Banaschewski, The real numbers in pointfree topology, Textos de Matemática. Série B [Texts in Mathematics. Series B], vol. 12, Universidade de Coimbra, Departamento de Matemática, Coimbra, 1997.

[4] - On the function ring functor in pointfree topology, Appl. Categ. Structures 13 (2005), no. 4, $305-328$.

[5] G. Bezhanishvili, N. Bezhanishvili, and J. Harding, Modal compact Hausdorff spaces, J. Logic Comput. 25 (2015), no. 1, 1-35.

[6] G. Bezhanishvili, L. Carai, and P.J. Morandi, Free bounded archimedean $\ell$-algebras, submitted. Available at arXiv:2008.01847, 2020.

[7] _ Modal operators on rings of continuous functions, submitted. Available at arXiv:1909.06912, 2020.

[8] G. Bezhanishvili, P. J. Morandi, and B. Olberding, Bounded Archimedean $\ell$-algebras and GelfandNeumark-Stone duality, Theory Appl. Categ. 28 (2013), Paper No. 16, 435-475. 
[9] _ A functional approach to Dedekind completions and the representation of vector lattices and

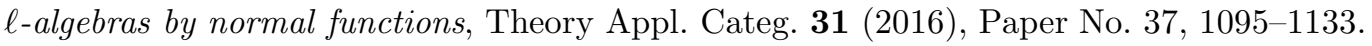

[10] G. Birkhoff, Lattice theory, third ed., American Mathematical Society Colloquium Publications, vol. 25, American Mathematical Society, Providence, R.I., 1979.

[11] A. Chagrov and M. Zakharyaschev, Modal logic, Oxford Logic Guides, vol. 35, The Clarendon Press, Oxford University Press, New York, 1997.

[12] R. Engelking, General topology, second ed., Sigma Series in Pure Mathematics, vol. 6, Heldermann Verlag, Berlin, 1989.

[13] L. L. Esakia, Topological Kripke models, Dokl. Akad. Nauk SSSR 214 (1974), 298-301.

[14] R. I. Goldblatt, Metamathematics of modal logic, Rep. Math. Logic (1976), no. 6, 41-77.

[15] P. R. Halmos, Algebraic logic. I. Monadic Boolean algebras, Compositio Math. 12 (1956), 217-249.

[16] M. Henriksen and D. G. Johnson, On the structure of a class of Archimedean lattice-ordered algebras, Fund. Math. 50 (1961/1962), 73-94.

[17] P. T. Johnstone, Stone spaces, Cambridge Studies in Advanced Mathematics, vol. 3, Cambridge University Press, Cambridge, 1982.

[18] B. Jónsson and A. Tarski, Boolean algebras with operators. I, Amer. J. Math. 73 (1951), 891-939.

[19] C. Kupke, A. Kurz, and Y. Venema, Stone coalgebras, Theoret. Comput. Sci. 327 (2004), no. 1-2, 109-134.

[20] S. Mac Lane, Categories for the working mathematician, Graduate Texts in Mathematics, Vol. 5, Springer-Verlag, New York, 1971.

[21] W. W. McGovern, Neat rings, J. Pure Appl. Algebra 205 (2006), no. 2, 243-265.

[22] Y. Venema, Algebras and coalgebras, Handbook of modal logic, vol. 3, Elsevier B. V., Amsterdam, 2007, pp. 331-426.

[23] K. Yosida, On vector lattice with a unit, Proc. Imp. Acad. Tokyo 17 (1941), 121-124.

Department of Mathematical Sciences, New Mexico State University, Las Cruces NM 88003 USA

Email address: guram@nmsu.edu, lcarai@nmsu.edu, pmorandi@nmsu.edu 